\title{
Serra de São Mamede Natural Park, a macrofungal diversity hotspot in the Mediterranean region
}

\author{
Celeste Santos-Silva ${ }^{1 *}$, Bruno Natário ${ }^{1}$, José Andrade $^{1}$, Rogério Louro ${ }^{1}$ \\ 1 Biology Department, Macromycology Laboratory, Mediterranean Institute for Agriculture, Environment and Development, University of Évora, \\ Évora, Portugal•CSS: css@uevora.pt (1ttps://orcid.org/0000-0002-2644-6639•BN: bafnatario@gmail.com•JA: jmcandrade21@gmail.com \\ •RL: rlouro@uevora.pt (1) https://orcid.org/0000-0002-4650-2669 \\ * Corresponding author
}

\begin{abstract}
The Serra de São Mamede Natural Park is a biodiversity hotspot in the broad region of Alentejo, Portugal, where the influence of the Atlantic Ocean allows for the continuity of a Eurosiberian-like forest in the heart of a Mediterranean region. The park is therefore unique among the few protected areas in Portugal. We attempt to describe the macrofungal diversity of the park for the first time. Our results reveal a rich and diverse assembly of macrofungal species (239 taxa), and in particular, mycorrhizal species (145 taxa). Furthermore, 26 taxa are newly recorded from Portugal, while 81 taxa are newly recorded from Alto Alentejo Province. Also, the majority of these newly reported taxa were recorded in mixed stands of Quercus pyrenaica Willd.-a European protected habitat of limited extent-whose importance is enhanced now the rich and diverse associated macrofungal community, as shown in our study.
\end{abstract}

\section{Keywords}

Ascomycota, Basidiomycota, biodiversity, Nature 2000, new records, Portugal, uncommon macrofungi

Academic editor: Panu Kunttu | Received 10 August 2021 | Accepted 19 January 2022 | Published 28 January 2022

Citation: Santos-Silva C, Natário B, Andrade J, Louro R (2022) Serra de São Mamede Natural Park, a macrofungal diversity hotspot in the Mediterranean region. Check List 18 (1): 109-137. https://doi.org/10.15560/18.1.109

\section{Introduction}

Besides the predominant Mediterranean climatic influence in mainland Portugal, there are also rare locations where the Atlantic influence supports the establishment and continuity of different plant communities. This is the case of São Mamede mountain range (Serra de São Mamede in Portuguese), located in Alentejo region. It is a biodiversity hotspot where the Atlantic influence allows the perpetuity of a Eurosiberian-like forest in the heart of a Mediterranean region. To ensure the conservation of its natural values and promote synergies with economic activities, Serra de São Mamede Natural Park (SSMNP) was established in 1989 and is now a Site of Community Importance (SCI) under Nature 2000 network (PTCON0007) (Oliveira 2014). Serra de São Mamede Natural Park represents the only natural reserve in northern Alentejo and is a unique and important ecosystem.

The topographic, edaphoclimatic, and geological features within the park area allows for a wide variety of habitats and species that make this park unique. SSMNP is one of the few protected areas in Portugal with a clear transition of ecosystems from north (Atlantic) to south (Mediterranean) (Marta-Pedroso et al. 2018), and this is reflected in the outstandingly rich flora $(\approx 900$ species $)$ 
(Flora-On 2022). The myriad of ecological niches present in the SSMNP - 21 natural and semi-natural habitats listed in Annex B-I of the Habitats Directive, including four priority habitats - harbour diverse plant communities. Many of its plant species are considered in the Habitats Directive: e.g., Ruscus aculeatus L; Narcissus pseudonarcissus L., and several members of Orchidaceae (ICNF 2003).

In the past, the original SSMNP Quercus pyrenaica Willd. forests that covered the majority of the area were gradually replaced with Castanea sativa Mill. groves and coppice stands. More recently, due to chestnut diseases and decreasing productivity, these $C$. sativa stands and shrublands areas have been replaced for more profitable plantations, such as Pinus pinaster Aiton and Eucalyptus spp. stands (Quintano et al. 2016). Also, native forests were removed for agriculture and pasture fields, putting Quercus pyrenaica forests and all their associated biodiversity at risk.

Given the SSMNP's unique location, rare bioclimatic combinations, and landscape mosaic which has been shaped by past human intervention, the park represents a remarkable location for examining and documenting the biological communities of these particular ecosystems. Although other biological groups in the park's ecosystems are well-documented (Castro-Antunes 1996; Malkmus 1997; Sérgio et al. 1997), the macrofungal communities are until now overlooked. Although several governmental agencies have stressed the urgency to address this knowledge gap and produce a macrofungal checklist for the park, the work was never done.

Therefore, we made an initial effort to characterise the macrofungal communities in SSMNP and provide its first macrofungal species checklist of this park.

\section{Study Area}

The survey was conducted in SSMNP, Alentejo, Portugal (Fig. 1), a Site of Community Importance under the Nature 2000 network (PTCON0007). The SSMNP, located between 350 and $1025 \mathrm{~m}$ above the sea level, is mainly comprised by the Luso-Spanish Serra de São Mamede which extends in a northwest to southeast direction for $40 \mathrm{~km}$ into Portuguese territory (ICNF 2003).

Granitic outcrops and quartzite ridges are the most remarkable orogenic features of SSMNP, and are accompanied, in the innermost area, by patches of schist and limestone (Costa et al. 1998; ICNF 2003). The Serra de São Mamede is the largest topographic feature south of the Tagus River and functions as a climate barrier. This mountain range protects the northern area of the park from the continental influences, enabling greater precipitation (800-1000 mm annually) and milder temperatures $\left(11.3{ }^{\circ} \mathrm{C}\right.$ median annual temperature) (Ferreira 2000). This is in contrast to the southeast slopes

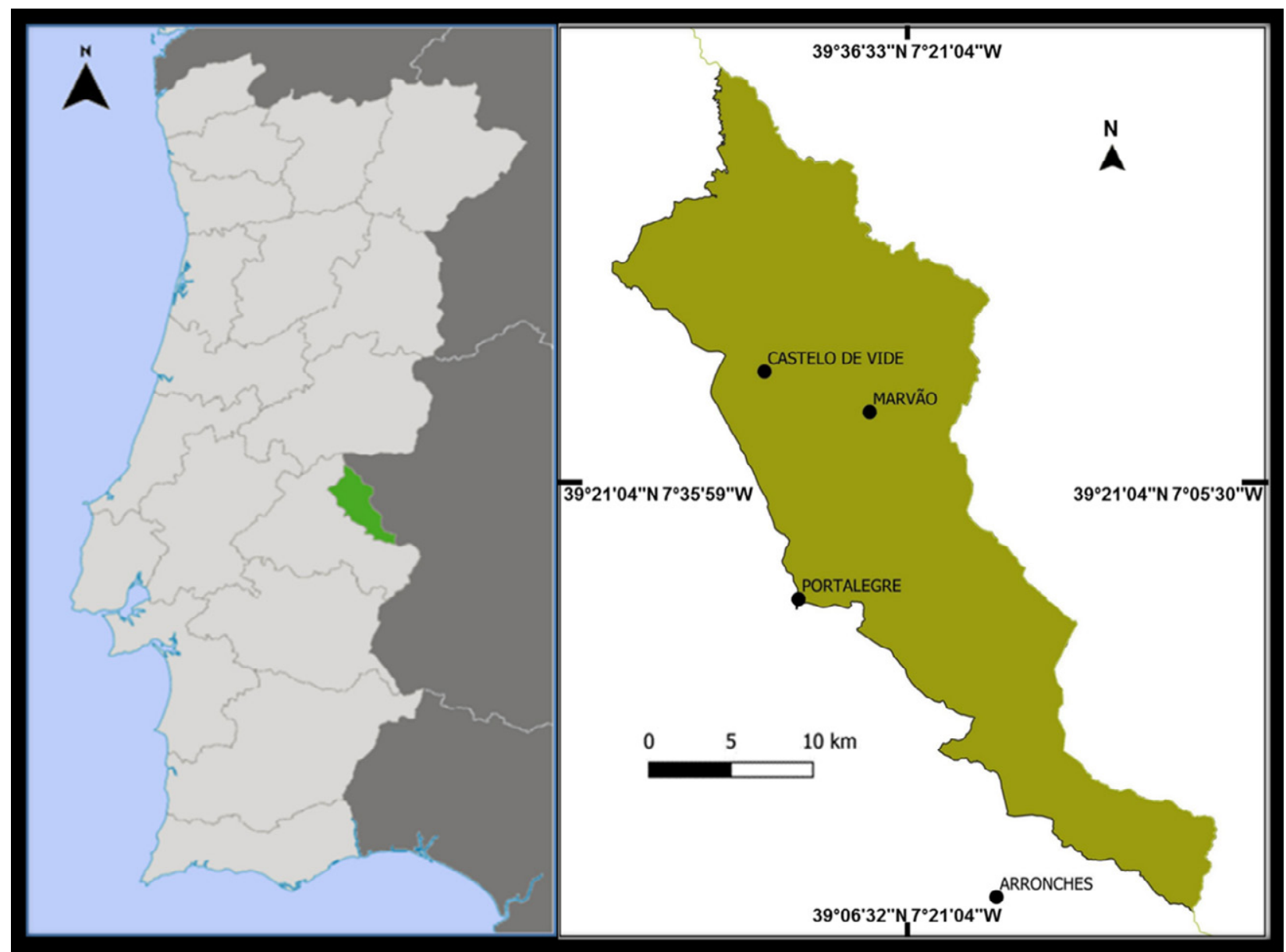

Figure 1. Location of SSMNP (green area) in Portugal (left); SSMNP (right). 
and plains in the south, which are exposed to continental winds having higher temperatures $\left(17.8{ }^{\circ} \mathrm{C}\right.$ median annual temperature) and less precipitation $(600-700 \mathrm{~mm}$ annually) (Ferreira 2000), and these areas thus exhibit a Mediterranean climate.

Forests of $Q$. pyrenaica (Annex B-I Habitats Directive, 9230) cover the granitic outcrops of the northern area, whereas downslope $Q$. pyrenaica stands form the unusual "montado/dehesa" landscape, which is unique in southern Portugal. The main aquatic feature of the park, the Sever River, is bordered by important alluvial forests of Alnus glutinosa (L.) Gaertn. and Fraxinus excelsior L. (Annex B-I Habitats Directive, 91B0), and of $F$. angustifolia subsp. angustifolia Vahl (Annex B-I Habitats Directive, 91E0). The southern part of the park is inhabited by Mediterranean species, mainly Quercus rotundifolia Lam. and Q. suber L. "montados". The heart of SSMNP is a transitory between the two bioclimates, where species typical of both climates co-exist, such as Castanea sativa (Annex B-I Habitats Directive, 9260), $Q$. suber L., and $Q$. pyrenaica. Other less prominent species, like $Q$. robur L., Q. lusitanica Lam., and Q. faginea Lam., prevail in smaller patches. Atlantic and Mediterranean shrub species-e.g., Pterospartum tridentatum (L.) Willk., Erica umbellata Loefl. ex L., Ulex minor Roth, Cytisus multiflorus (L'Hér.) Sweet, Cistus ladanifer L., and Arbutus unedo L.--follow the same pattern as tree species, occurring in somewhat limited areas from north to south (ICNF 2003).

The unique bioclimatic conditions, rich flora, and contrasting landscape heterogeneity allows for a wide variety of faunal groups to thrive within the park. Notably, the park is home to about 150 bird species, among which, Aquila fasciata (Vieillot, 1822), the symbol of the park, Aquila adalberti (C.L. Brehm, 1861), and Neophron percnopterus (Linnaeus, 1758), are categorized as Endangered or Critically Endangered (Cabral et al. 2005). Also impressive is the herpetological fauna of the park, with the presence of 15 of the 17 known species of amphibians in Portugal and 20 of the 35 species of reptiles occurring in the country.

Another biological group remarkably well represented in the SSMNP is the Chiroptera, with 19 species, including eight species of conservation concern (Cabral et al. 2005). The SSMNP harbours a few national and Iberian endemics as well, such as Anaecypris hispanica (Steindachner, 1866), Luciobarbus comizo (Steindachner, 1864), Alytes cisternasii (Boscá, 1879), Rana iberica (Boulenger, 1879), and Microtus cabrerae (Thomas, 1906) (ICNF 2003; Cabral et al. 2005).

\section{Methods}

We conducted fortnightly macrofungal surveys between October 2013 and April 2020, with sampling done in autumn to winter (October to March) and spring (March to June), the most favorable seasons. We sampled in several areas with distinct soil types: broadleaf stands, coniferous stands, and shrublands. Within each sampling site, the dominant or co-dominant trees were recorded. Macrofungal specimens were harvested, stored below $4{ }^{\circ} \mathrm{C}$ and processed within $24 \mathrm{~h}$. Morphologic identification-macroscopic, microscopic, and organoleptic features-of collected specimens, their putative trophic group, and their biogeographical preference were based on keys, monographs, and field guides (e.g., Breitenbach and Kränzlin 1981, 1986, 1991, 1995, 2000; Pegler et al. 1995; Calonge 1998; Gerhardt et al. 2000; Frade and Alfonso 2003; Bon 2004; Kränzlin 2005; Sarnari 1998, 2007; Roux 2006; Moreno 2010). These specimens were then preserved and deposited in Évora University herbarium (UEVH- FUNGI).

Macrofungi nomenclature follows Index Fungorum (http://www.indexfungorum.org/; accessed on: 2022-120). Current geographic ranges of species were found an unpublished (2002) preliminary list of agaricoid fungi of Portugal, Natário et al. (2019), GBIF (2021), and other regional publications. We follow Calonge (1998) in our division of Iberian territory into provinces.

Macrofungal species are arranged alphabetically, according to the higher taxonomic placement (phylum, family). First records for continental Portugal (N) and first records for Alto Alentejo Province (n) are highlighted. Several features are also described for each species: a) the putative trophic group (M: mycorrhizal, $\mathbf{S}$ : saprotrophic or $\mathbf{P}$ : parasitic); b) the forest stand were they most frequently occurred, with indication of the dominant (co-dominant) tree species (Qp/Qs: Q. pyrenaica and Q. suber, Qp/Cs: Q. pyrenaica and C. sativa, Qs/ Qr: $Q$. suber and $Q$. rotundifolia, Fa: Fraxinus angustifolia Vahl., Ppi: P. pinaster, Ppa: Populus alba L., Ppn: Populus nigra L. and S: shrublands); c) species biogeographical preference (Atl: Atlantic, Med: Mediterranean, or M/A: indifferent); d) the UEVH-FUNGI code (Herbarium accession numbers).

\section{Results}

A total of 239 macrofungal species, representing 53 families and 112 genera, were recorded in this study. The majority of the taxa belong to Basidiomycota (91\%), and only $9 \%$ to Ascomycota. The most represented of Basidiomycota were Russulaceae (38 spp.), Tricholomataceae (22 spp.), and Boletaceae (21 spp.), accounting for 37\% of all Basidiomycota species sampled (Fig. 2), which is not surprising, as these are well-known specious families. Considering the trophic groups, mycorrhizal species (144) greatly outnumber saprotrophic taxa (90) while only five parasitic species were identified. This outcome mirrors the host diversity present in the region as most taxa have an ectomycorrhizal lifestyle.

As for novelties, 26 recorded species were newly found in Portugal (all belonging to Basidiomycota); 60\% of them are mycorrhizal and $40 \%$ are saprotrophic. Furthermore, 81 species were found to be new records for Alto Alentejo Province, with a mycorrhizal/saprotrophic 


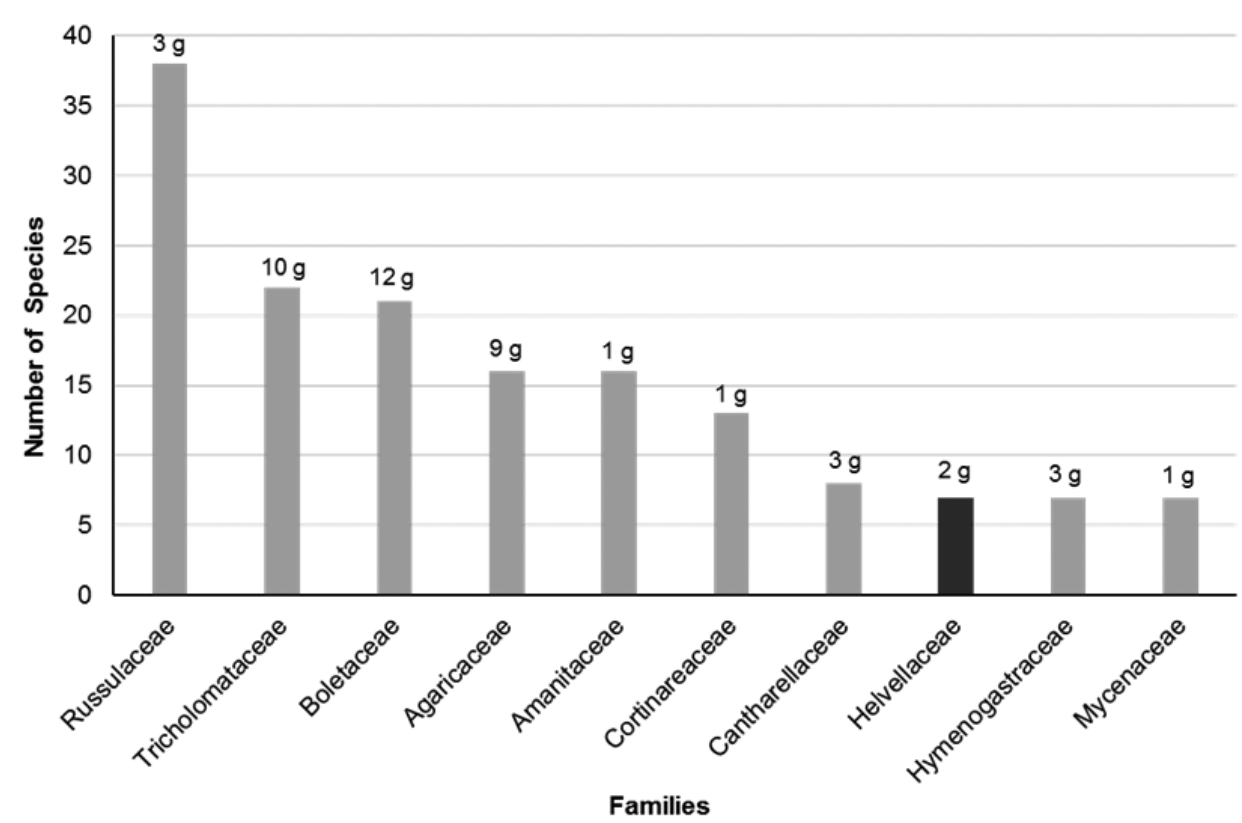

Figure 2. Number of species of the 10 most numerous families and number of genera ( $\mathrm{g}$ ) in each family. Grey bars = Basidiomycota; the black bar = Ascomycota.

ratio similar to the former, plus two parasitic taxa. Of the taxa newly recorded from Alto Alentejo Province, 15 belong to Ascomycota and 66 belong to Basidiomycota. About $77 \%$ of the taxa newly recorded from Portugal and $68 \%$ of the taxa newly recorded from Alto Alentejo occurred in stands of $Q$. pyrenaica mixed with $Q$. suber or C. sativa. Nearly $70 \%$ of the total identified species have a wide biogeographical range, $19 \%$ are specific to Mediterranean areas, and $11 \%$ are specific to Atlantic regions. Nearly half of the identified species occurred preferentially in areas with mixed stands of $Q$. pyrenaica/ $Q$. suber, while approximately a quarter of the taxa preferentially occurred in mixed stands of $Q$. pyrenaica/C. sativa. Pinus pinaster stands and $Q$. suber/Q. rotundifolia mixed stands were less species rich than Q. pyrenaica mixed stands. Only five macrofungal species were more frequently found in shrublands, while14 species occurred more frequently in riparian areas, dominated by $P$. nigra, P. alba, or F. angustifolia.

\section{ASCOMYCOTA}

Cordycipitaceae

Cordyceps militaris (L.) Fr.

Figure $3 \mathrm{~A}$

Material examined. Newly recorded (n). PORTUGAL - Alentejo • SSMNP; 39 $18^{\prime} 06^{\prime \prime} \mathrm{N}, 007^{\circ} 24^{\prime} 10^{\prime \prime} \mathrm{W}$; alt. 700 m; 27.III.2018; Andrade J. leg.; P (parasitic of Lepidoptera species); Ppi; Atl; UEVH-FUNGI 2003994.

Identification. This species can be recognized by the club-shaped ascocarps measuring 1-6 cm. The fertile head is bright orange to reddish with a rough or granular texture, due to semi-immersed perithecia. The unfertile stipe is slightly sinuous, yellowish to light orange, paler than the fertile head. Perithecia broadly ovoid.
Asci broadly ellipsoid, 8-spored. Ascospores multiseptated, filiform, smooth and hyaline; breaking into ellipsoidal part-spore segments. This species can be easily distinguished microscopically from any other Cordycipitaceae, like Blackwellomyces cardinalis (G.H. Sung \& Spatafora) Spatafora \& Luangsa-ard and B. pseudomilitaris (Hywel-Jones \& Sivichai) Spatafora \& Luangsaard, by its disarticulating ascospores.

\section{Geoglossaceae}

\section{Trichoglossum hirsutum (Pers.) Boud.}

Material examined. Newly recorded (n). PORTUGAL - Alentejo - SSMNP; $39^{\circ} 08^{\prime} 15^{\prime \prime} \mathrm{N}, 007^{\circ} 12^{\prime} 54^{\prime \prime} \mathrm{W}$; alt. 370 m; 20.II.2020; Andrade J. leg.; S; Qs/Qr; Med; UEVHFUNGI 2004831.

Helvellaceae

Dissingia leucomelaena (Pers.) K. Hansen \& X.H. Wang

Material examined. Newly recorded (n). PORTUGAL - Alentejo - SSMNP; 39 $17^{\prime} 10^{\prime \prime} \mathrm{N}, 007^{\circ} 20^{\prime} 57^{\prime \prime} \mathrm{W}$; alt. 770 m; 30.I.2016; Andrade J. leg.; M; Ppi; M/A; UEVHFUNGI 2003365.

\section{Helvella acetabulum (L.) Quél.}

Figure 3B

Material examined. Newly recorded (n). PORTUGAL - Alentejo • SSMNP; 39 $18^{\prime} 15^{\prime \prime} \mathrm{N}, 007^{\circ} 24^{\prime} 35^{\prime \prime} \mathrm{W}$; alt. 720 m; 01.III.2020; Andrade J. leg.; M; Qp/Cs; M/A; UEVHFUNGI 2004833.

Identification. This species can be recognized by the cup-shaped ascocarp, 2-9 $\mathrm{cm}$ in diameter. Pileus upper surface yellow-brown to brown, smooth; under surface brown to yellow-brown, sometimes paler near the stipe. 


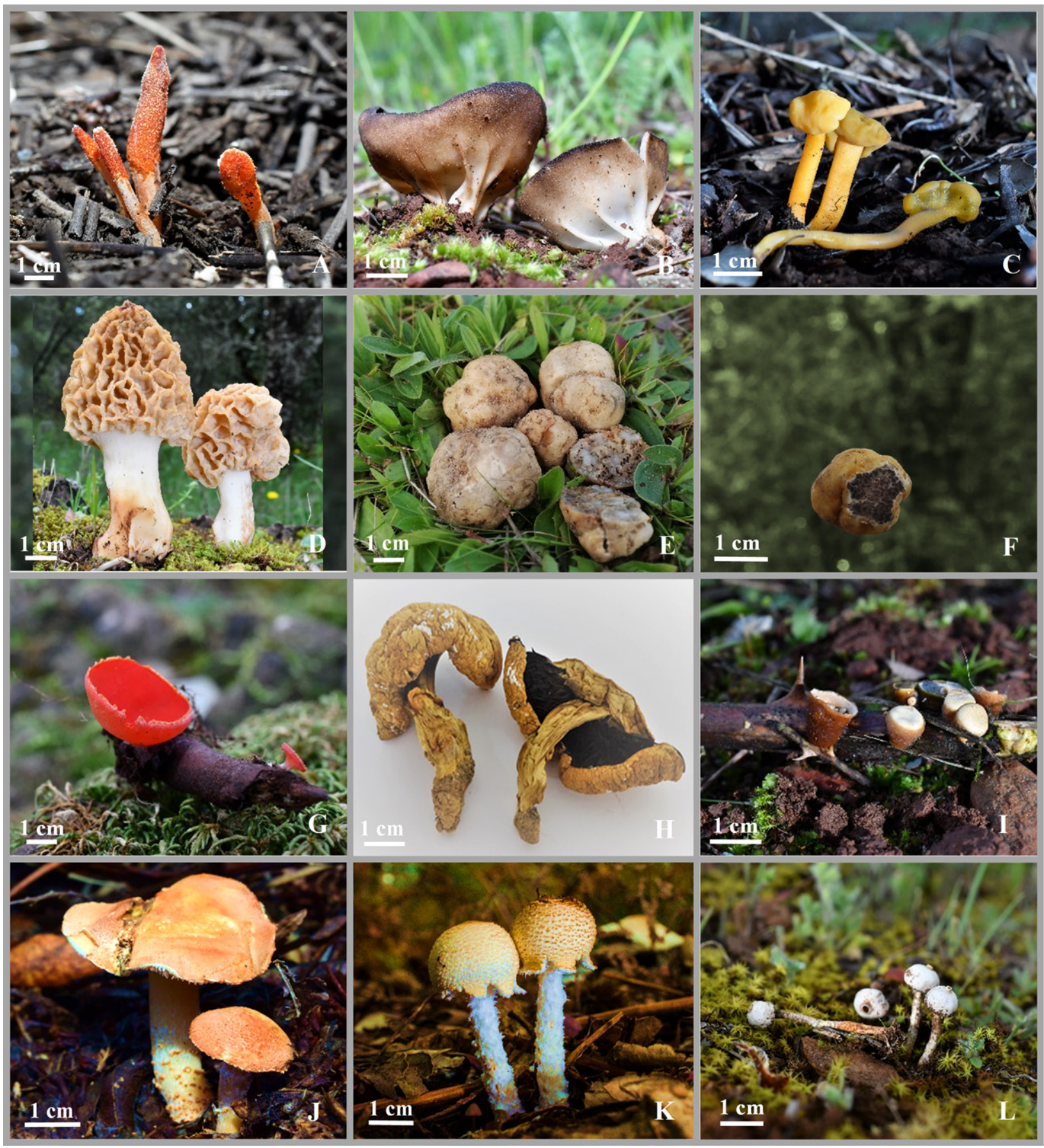

Figure 3. A. Cordyceps militaris (L.) Fr.* B. Helvella acetabulum (L.) Quél.* C. Leotia lubrica (Scop.) Pers. * D. Morchella esculenta (L.) Pers.* E. Terfezia extremadurensis Muñ.-Moh., Ant. Rodr. \& Bordallo* F. Terfezia pini Bordallo, Ant.Rodr. \& Mohedano* G. Sarcoscypha coccinea (Gray) Boud.* H. Agaricus lutosus F.H. Møller.** I. Crucibulum laeve (Huds.) Kambly* J. Cystodermella terryi (Berk. \& Broome) Bellù.** K. Lepiota magnispora Murrill.** L. Tulostoma brumale Pers.* ${ }^{*}=$ newly recorded from Alto Alentejo Province; ${ }^{* *}=$ newly recorded from continental Portugal)

Stipe whitish to brownish, finely hairy, deeply pocketed and ribbed; with sharp-edged forking ribs that extend onto the under surface of the pileus. Spores 16-20×11-14 $\mu \mathrm{m}$, elliptical, smooth, with one central oil droplet. This relatively large cup-shaped fungus is characterized by a brownish ascocarp with prominent branching ribs, resembling a cabbage leaf. It can be confused with $\mathrm{H}$. leucomelaena (Pers.) Nannf., which has a darker coloured pileus and a short stipe without ribs, and with Helvella queletii Bres., which has a well-developed ribbed stipe, but the ribs seldom extend past the base of the cup.

\section{Helvella atra J. König}

Material examined. PORTUGAL - Alentejo - SSMNP; $39^{\circ} 18^{\prime} 15^{\prime \prime} \mathrm{N}, 007^{\circ} 24^{\prime} 35^{\prime \prime} \mathrm{W}$; alt. $720 \mathrm{~m}$; 03.I.2016; Andrade J. leg.; M; Qp/Cs; M/A; UEVH-FUNGI 2003366.

\section{Helvella crispa (Scop.) Fr.}

Material examined. Newly recorded (n). PORTUGAL - Alentejo • SSMNP; 39²0'03"N, 007²4'33"W; alt. 690 m; 30.I.2016; Andrade J. leg.; M; Qp/Cs; Atl.; UEVHFUNGI 2003364. 


\section{Helvella elastica Bull.}

Material examined. Newly recorded (n). PORTUGAL -Alentejo - SSMNP; 39²7'12"N, 007²6'33"W; alt. 440 m; 03.II.2020; Andrade J. leg.; M; Qp/Cs; Med.; UEVHFUNGI 2004828.

\section{Helvella lacunosa Afzel.}

Material examined. PORTUGAL - Alentejo • SSMNP; $39^{\circ} 23^{\prime} 56^{\prime \prime} \mathrm{N}, 007^{\circ} 25^{\prime} 34^{\prime \prime}$ Walt. 580 m; 08.I.2014; Andrade J. leg.; M; Qp/Qs; M/A; UEVH-FUNGI 2002754.

\section{Helvella macropus (Pers.) P. Karst.}

Material examined. Newly recorded (n). PORTUGAL - Alentejo - SSMNP; 39²7'12"N, 007²6'33"W; alt. 440 m; 24.II.2014; Andrade J. leg.; M; Qp/Cs; M/A; UEVHFUNGI 2002768.

\section{Leotiaceae}

\section{Leotia lubrica (Scop.) Pers.}

Figure 3C

Material examined. Newly recorded (n). PORTUGAL - Alentejo - SSMNP; 39 $18^{\prime} 05^{\prime \prime} \mathrm{N}, 007^{\circ} 24^{\prime} 40^{\prime \prime} \mathrm{W}$; alt. 700 m; 02.II.2020; Andrade J. leg.; S; Qp/Cs; M/A; UEVHFUNGI 2004782.

Identification. This species can be recognized by the viscid, yellowish or olive ochre pileus, with curved, lobed margin. Viscid hymenium concolourous with the pileus, viscid yellowish stipe, and gelatinous flesh. Asci non amyloid and 8-spored. Ascospores fusiform, smooth, hyaline and multiseptate (5-7 septa). It can be distinguished from the closely resembling Leotia atrovirens Pers. by its yellowish or olive-ochre colour and multiseptate ascospores.

Morchellaceae

\section{Morchella esculenta (L.) Pers.}

Figure 3D

Material examined. Newly recorded (n). PORTUGAL -Alentejo - SSMNP; 39³0'24"N, 007²6'01"W; alt. 280 m; 10.IV.2020; Andrade J. leg.; S; Ppa; M/A; UEVHFUNGI 2004908.

Identification. This species can be recognized by the oval to globose pileus, with several irregularly distributed pits, separated by narrow ridges. Ascocarp measuring up to $12 \mathrm{~cm}$, pale cream to light brown, with the ridges usually lighter colour than the pits. Pileus margin curved and attached to the stipe. Stipe cylindrical or tapering near the apex, hollow, ranging from white to pale cream. Asci cylindrical, hyaline, and 8-spored. Ascospores ellipsoidal, smooth, and hyaline. It can be distinguished from the similar Morchella elata Fr., which has a darker, more or less conic pileus.

\section{Pezizaceae}

\section{Peziza saccardoana Cooke}

Material examined. Newly recorded (n). PORTUGAL - Alentejo • SSMNP; $39^{\circ} 27^{\prime} 12^{\prime \prime} \mathrm{N}, 007^{\circ} 26^{\prime} 33^{\prime \prime} \mathrm{W}$; alt. 440 m; 10.VI.2018; Andrade J. leg.; M; Qp/Qs; M/A; UEVHFUNGI 2004139.

\section{Terfezia arenaria (Moris) Trappe}

Material examined. PORTUGAL - Alentejo - SSMNP; $39^{\circ} 23^{\prime} 56^{\prime \prime} \mathrm{N}, 007^{\circ} 25^{\prime} 34^{\prime \prime} \mathrm{W}$; alt. $580 \mathrm{~m}$; 28.III.2016; Andrade J. leg.; M; Qp/Qs; M/A; UEVH-FUNGI 2003451.

\section{Terfezia extremadurensis Muñ.-Moh., Ant. Rodr. \&} Bordallo

Figure 3E

Material examined. Newly recorded (n). PORTUGAL - Alentejo • SSMNP; 39²3'56"N, 007²5'34"W; alt. 580 m; 14.II.2019; Andrade J. leg.; M; Qp/Qs; M/A; UEVHFUNGI 2004567.

Identification. Ascocarp hypogeous to partially emergent, 2-5 cm in diameter, subglobose, nodulose, furrowed, and often with a small basal depression; first cream coloured, then turning brown and often with black spots on sun-exposed parts. Peridium 300-600 $\mu \mathrm{m}$ thick, concolourous with the external surface in cross section. Gleba solid, fleshy, initially whitish then salmon pink, with meandering veins not surrounding the fertile tissue and not forming pockets; darkening with age and becoming greenish grey. Asci randomly arranged in the gleba, nonamyloid, subglobose to ovate, sessile or short-stipitate. Ascospores globose, yellowish, and ornamented with conical blunt and occasionally truncated spines, 3-5 $\mu \mathrm{m}$ long and 1-3 $\mu \mathrm{m}$ wide at the base. This species can be distinguished from other Terfezia species by its larger spores with wider spines and its tuber-like glebal morphology.

\section{Terfezia fanfanii Mattir.}

Material examined. PORTUGAL - Alentejo - SSMNP; $39^{\circ} 23^{\prime} 56^{\prime \prime} \mathrm{N}, 007^{\circ} 25^{\prime} 34^{\prime \prime} \mathrm{W}$; alt. $580 \mathrm{~m}$; 14.II.2019; Andrade J. leg.; M; Qp/Qs; M/A; UEVH-FUNGI 2004577.

\section{Terfezia pini Bordallo, Ant.Rodr. \& Mohedano}

Figure $3 \mathrm{~F}$

Material examined. Newly recorded (n). PORTUGAL - Alentejo • SSMNP; $39^{\circ} 08^{\prime} 04^{\prime \prime} \mathrm{N}, 007^{\circ} 12^{\prime} 37^{\prime \prime} \mathrm{W}$; alt. 370 m; 14.II.2019; Andrade J. leg.; M; Qs/Qr; M/A; UEVHFUNGI 2004594.

Identification. Ascocarp hypogeous to partially emergent, 1-2 cm in diameter, subglobose; rounded sterile base with a mycelial tuft; ranging from cream colour to light ochre upon maturity, smooth to touch. Peridium 300-600 $\mu \mathrm{m}$ thick, poorly delimited, pseudoparenchymatous, composed by thick-walled subglobose cells. Gleba solid, fleshy, initially whitish then with brownish or greenish pockets of fertile tissue, separated by whitish sterile veins. Asci randomly arranged in the gleba, 
nonamyloid, subglobose to ovate, sessile or short-stipitate, 6-8-spored. Ascospores globose, yellow ochre, and ornamented with conical, sometimes finger-like, flexuous, blunt spines, 3-4(-5) $\mu \mathrm{m}$ long, 1-2 $\mu \mathrm{m}$ wide at the base, occasionally interconnected forming a pseudoreticulum. The small ascocarps, different habitat, and spore morphology distinguish this species from T. fanfani Mattir.

\section{Pyronemataceae}

\section{Otidea bufonia (Pers.) Boud.}

Material examined. PORTUGAL - Alentejo • SSMNP; $39^{\circ} 19^{\prime} 17^{\prime \prime} \mathrm{N}, 007^{\circ} 23^{\prime} 08^{\prime \prime} \mathrm{W}$; alt. 770 m; 6.XII.2019; Andrade J. leg.; M; Ppi; M/A; UEVH-FUNGI 2004783.

\section{Rutstroemiaceae}

\section{Lanzia echinophila (Bull.) Korf}

Material examined. Newly recorded (n). PORTUGAL - Alentejo - SSMNP; $39^{\circ} 24^{\prime} 23^{\prime \prime} \mathrm{N}, 007^{\circ} 24^{\prime} 31^{\prime \prime} \mathrm{W}$; alt. 660 m; 12.XI.2016; Andrade J. leg.; S; Qp/Cs; M/A; UEVHFUNGI 2003608.

\section{Rutstroemia firma (Pers.) P. Karst.}

Material examined. Newly recorded (n). PORTUGAL - Alentejo • SSMNP; 39 $18^{\prime} 15^{\prime \prime} \mathrm{N}, 007^{\circ} 24^{\prime} 35^{\prime \prime} \mathrm{W}$; alt. 720 m; 12.XI.2016; Andrade J. leg.; S; Qp/Cs; Atl.; UEVHFUNGI 2003894.

Sarcoscyphaceae

\section{Sarcoscypha coccinea (Gray) Boud.}

Figure $3 \mathrm{G}$

Material examined. Newly recorded (n). PORTUGAL - Alentejo - SSMNP; 39 $18^{\prime} 03^{\prime \prime} \mathrm{N}, 007^{\circ} 24^{\prime} 43^{\prime \prime} \mathrm{W}$; alt. 680 m; 3.II.2020; Andrade J. leg.; S; Qp/Qs; M/A; UEVHFUNGI 2004829.

Identification. This species can be recognized by the irregular cup-like ascocarps, measuring up to $5 \mathrm{~cm}$. Hymenial inner surface smooth, bright pink to scarlet red. Margin usually incurved, whitish. Outer surface of the hymenium paler than the inside; tomentose, covered with straight or bent hairs. With a short stipe-like structure, often buried in moss. Asci cylindrical and 8-spored. Spores elongated or ellipsoidal smooth, hyaline, 26-40 × 10-12 $\mu \mathrm{m}$. This species can be distinguished from Sarcoscypha austriaca (Beck ex Sacc.) Boud., also a bright red, cuplike Sarcoscyphaceae, by the straight hairs (tomentum) instead of coiled hairs and the absence of spores with conidial bud protrusions.

\section{Xylariaceae}

\section{Poronia punctata (L.) Fr.}

Material examined. PORTUGAL - Alentejo - SSMNP; $39^{\circ} 27^{\prime} 18^{\prime \prime} \mathrm{N}, 007^{\circ} 26^{\prime} 35^{\prime \prime} \mathrm{W}$; alt. 430 m; 29.XII.2013; Andrade J. leg.; S; Qs/Qr; M/A; UEVH-FUNGI 2001791.

\section{BASIDIOMYCOTA}

Agaricaceae

Agaricus arvensis Schaeff.

Material examined. PORTUGAL - Alentejo • SSMNP; $39^{\circ} 23^{\prime} 56^{\prime \prime} \mathrm{N}, 007^{\circ} 25^{\prime} 34^{\prime \prime} \mathrm{W}$; alt. $580 \mathrm{~m}$; 12.XII.2013; Andrade J. leg.; S; Qp/Qs; Atl.; UEVH-FUNGI 2002409.

\section{Agaricus augustus Fr.}

Material examined. PORTUGAL - Alentejo - SSMNP; $39^{\circ} 10^{\prime} 11^{\prime \prime} \mathrm{N}, 007^{\circ} 09^{\prime} 37^{\prime \prime} \mathrm{W}$; alt. $350 \mathrm{~m}$; 30.XI.2015; Andrade J. leg.; S; Qs/Qr; Med.; UEVH-FUNGI 2003420.

\section{Agaricus campestris L.}

Material examined. PORTUGAL - Alentejo - SSMNP; $39^{\circ} 19^{\prime} 17^{\prime \prime} \mathrm{N}, 007^{\circ} 24^{\prime} 54^{\prime \prime} \mathrm{W}$; alt. 600 m; 03.XII.2013; Andrade J. leg.; S; Qp/Cs; M/A; UEVH-FUNGI 2002416.

\section{Agaricus lutosus F.H. Møller}

Figure $3 \mathrm{H}$

Material examined. Newly recorded (n). PORTUGAL - Alentejo • SSMNP; 39²3'19"N, 007²5'21"W; alt. 620 m; 26.XI.2019; Andrade J. leg.; S; Qp/Cs; M/A; UEVHFUNGI 2004804.

Identification. This species can be recognized by the hemispheric to slightly convex pileus, covered by fibrillose brownish to purplish scales, darker in the centre of the pileus. Free pale pinkish-grey lamellae, turning reddish-brown and finally dark brown. Short, clavate, whitish stipe with an apical, simple, fleeting ring. Yellowing (pileus, stipe, ring, and flesh) with time or when bruised. Faint smell of bitter almonds. Species belong to section Minores, with spores on average as long as or shorter than $5.4 \mu \mathrm{m}$ and without cheilocystidia. Agaricus pseudolutosus (G. Moreno, Esteve-Rav., Illana \& Heykoop) G. Moreno, L.A. Parra, Esteve-Rav. \& Heykoop has a more intense yellowing reaction when bruised and presents microscopic differences.

\section{Agaricus sylvicola (Vittad.) Peck}

Material examined. Newly recorded (n). PORTUGAL - Alentejo • SSMNP; 39 $08^{\prime} 04^{\prime \prime} \mathrm{N}, 007^{\circ} 12^{\prime} 37^{\prime \prime} \mathrm{W}$; alt. 370 m; 11.XI.2018; Andrade J. leg.; S; Qs/Qr; Med.; UEVHFUNGI 2004315.

\section{Coprinus comatus (O. F. Müll.) Pers.}

Material examined. PORTUGAL - Alentejo - SSMNP; $39^{\circ} 22^{\prime} 48^{\prime \prime} \mathrm{N}, 007^{\circ} 23^{\prime} 11^{\prime \prime} \mathrm{W}$; alt. 520 m; 09.XI.2013; Andrade J. leg.; S; Ppn; Med.; UEVH-FUNGI 2002662.

\section{Crucibulum laeve (Huds.) Kambly}

Figure 3I

Material examined. Newly recorded (n). PORTUGAL - Alentejo • SSMNP; 39²3'19"N, 007²5'21"W; alt. 620 m; 05.XI.2016; Andrade J. leg.; S (on dead branches of $C$. sativa); Qp/Cs; M/A; UEVH-FUNGI 2003579.

Identification. This species can be recognized by the 
small cup- or crucible-shaped basidiocarp, growing to 1 $\mathrm{cm}$. Epiphragm yellow, smooth and velvety, covering the top of the single-layered peridium when immature and breaking up at maturity to expose up to eight peridioles. The outer surface of the peridium brownish, initially fibrillose then smooth; the inner surface of the peridium is smooth, pale grey to light-brown. Peridioles flattened, whitish, 1-2 $\mathrm{mm}$ broad and connected to the peridium by a thin cord (funiculus). This species can be easily differentiated from Cyathus striatus (Huds.) Willd, by the single layered peridium, smooth inner surface of the peridium and whitish peridioles.

\section{Cyathus striatus (Huds.) Willd.}

Material examined. Newly recorded (n). PORTUGAL - Alentejo - SSMNP; 39²4'23"N, 007²4'31"W; alt. 660 m; 20.X.2019; Andrade J. leg.; S; Qp/Cs; M/A; UEVHFUNGI 2004761.

\section{Cystodermella terryi (Berk. \& Broome) Bellù}

Figure 3J

Material examined. Newly recorded (n). PORTUGAL - Alentejo • SSMNP; $39^{\circ} 18^{\prime} 15^{\prime \prime} \mathrm{N}, 007^{\circ} 24^{\prime} 35^{\prime \prime} \mathrm{W}$; alt. 720 m; 20.XII.2017; Andrade J. leg.; S; Qp/Cs; M/A; UEVHFUNGI 2003965.

Identification. This species can be recognized by the brick-red hemispherical to plano-convex granulose pileus, whitish lamellae, and stipe covered with flaky bracelets, concolourous with the pileus below the ring and white and smooth above the ring. Microscopically, the oval, hyaline, non-amyloid spores $(4.3 \times 2.7 \mu \mathrm{m})$ and abundant urticiform cheilocystidia are the main distinctive features. This species can be distinguished from the similar Cystoderma amianthinum (Scop.) Fayod by the non-amyloid spores, and from Cystodermella adnatifolia (Peck) Harmaja by the presence of cystidia.

\section{Lepiota castanea Quél.}

Material examined. PORTUGAL - Alentejo • SSMNP; $39^{\circ} 27^{\prime} 12^{\prime \prime} \mathrm{N}, 007^{\circ} 26^{\prime} 33^{\prime \prime} \mathrm{W}$; alt. 440 m; 12.XI.2016; Andrade J. leg.; S; Qp/Qs; M/A; UEVH-FUNGI 2003606.

Lepiota clypeolaria (Bull.) P. Kumm.

Material examined. PORTUGAL - Alentejo • SSMNP; $39^{\circ} 27^{\prime} 12^{\prime \prime} \mathrm{N}, 007^{\circ} 26^{\prime} 33^{\prime \prime} \mathrm{W}$; alt. 440 m; 02.X.2014; Andrade J. leg.; S; Qp/Qs; M/A; UEVH-FUNGI 2002951.

\section{Lepiota magnispora Murrill}

Figure $3 \mathrm{~K}$

Material examined. Newly recorded (n). PORTUGAL - Alentejo • SSMNP; 39 $18^{\prime} 15^{\prime \prime} \mathrm{N}, 007^{\circ} 24^{\prime} 35^{\prime \prime} \mathrm{W}$; alt. 720 m; 04.XI.2018; Andrade J. leg.; S; Qp/Cs; M/A; UEVHFUNGI 2004476.

Identification. This species can be recognized by the hemispherical to plano-convex pileus with a reddish-orange, smooth centre, surrounded by concentric rings of reddish-orange scales, becoming yellowish-orange towards the margin. Free, white to cream lamellae. Stipe finely woolly above the ring zone (often poorly delineated) and covered by large yellow or yellow-orange woolly scales below the ring zone. Large, $17 \times 5 \mu \mathrm{m}$, ellipsoidal to ovoid spores. This species can be distinguished from the similar L. ignivolvata Bousset \& Joss. ex Joss. and L. clypeolaria (Bull.) P. Kumm. by the yellowish-orange hues on the stipe and pileus and by the larger spores.

\section{Lycoperdon atropurpureum Vittad.}

Material examined. PORTUGAL - Alentejo • SSMNP; $39^{\circ} 28^{\prime} 11^{\prime \prime} \mathrm{N}, 007^{\circ} 25^{\prime} 04^{\prime \prime} \mathrm{W}$; alt. 400 m; 02.X.2014; Andrade J. leg.; S; Qs/Qr; Med.; UEVH-FUNGI 2002882.

\section{Lycoperdon perlatum Pers.}

Material examined. PORTUGAL - Alentejo - SSMNP; $39^{\circ} 19^{\prime} 15^{\prime \prime} \mathrm{N}, 007^{\circ} 24^{\prime} 47^{\prime \prime} \mathrm{W}$; alt. 600 m; 19.I.2016; Andrade J. leg.; S; Qp/Qs; M/A.; UEVH-FUNGI 2003332.

\section{Macrolepiota procera (Scop.) Singer}

Material examined. PORTUGAL - Alentejo - SSMNP; $39^{\circ} 22^{\prime} 54^{\prime \prime} \mathrm{N}, 007^{\circ} 23^{\prime} 31^{\prime \prime} \mathrm{W}$; alt. 520 m; 24.XI.2015; Andrade J. leg.; S; Fa; M/A.; UEVH-FUNGI 2003044.

\section{Tulostoma brumale Pers.}

Figure 3L

Material examined. Newly recorded (n). PORTUGAL - Alentejo - SSMNP; 39 29'45"N, 007²7'03"W; alt. 370 m; 18.III.2016; Andrade J. leg.; S; Qs/Qr; Med.; UEVHFUNGI 2003388.

Identification. This species can be recognized by stipitate, gasteroid basidiocarps with a globose head, 0.5-1 $\mathrm{cm}$ in diameter. Peridium smooth to granular, varying from whitish to greyish, with a protruding peristome (opening) delimited by a reddish ring. Gleba is compact, white at first, then turning powdery brown at maturity. Stipe is fibrous and mottled grey-brown. Spores subspherical to globose, $4-5 \times 3.5-4 \mu \mathrm{m}$, covered in fine warts. It can be distinguished from Tulostoma melanocyclum Bres. by the smaller spores and from T. niveum Kers by the less prominent peristome.

Amanitaceae

\section{Amanita badia (Schaeff.) Bon \& Contu}

Material examined. Newly recorded (n). PORTUGAL - Alentejo • SSMNP; 39 $19^{\prime} 17^{\prime \prime} \mathrm{N}, 007^{\circ} 24^{\prime} 54^{\prime \prime} \mathrm{W}$; alt. 600 m; 26.XI.2019; Andrade J. leg.; M; Qp/Cs; M/A; UEVHFUNGI 2004805.

\section{Amanita battarrae (Boud.) Bon}

Material examined. PORTUGAL - Alentejo - SSMNP; $39^{\circ} 19^{\prime} 17^{\prime \prime} \mathrm{N}, 007^{\circ} 24^{\prime} 54^{\prime \prime} \mathrm{W}$; alt. 600 m; 16.XI.2018; Andrade J. leg.; M; Qp/Cs; M/A; UEVH-FUNGI 2004330. Amanita caesarea (Scop.) Pers.

Material examined. PORTUGAL - Alentejo - SSMNP; $39^{\circ} 18^{\prime} 05^{\prime \prime} \mathrm{N}, 007^{\circ} 24^{\prime} 40^{\prime \prime} \mathrm{W}$; alt. 700 m; 24.XI.2015; Andrade J. leg.; M; Qp/Cs; M/A; UEVH-FUNGI 2003029. 
Amanita citrina Pers.

Material examined. PORTUGAL - Alentejo • SSMNP; $39^{\circ} 17^{\prime} 15^{\prime \prime} \mathrm{N}, 007^{\circ} 21^{\prime} 18^{\prime \prime} \mathrm{W}$; alt. $770 \mathrm{~m}$; 05.XII.2016; Andrade J. leg.; M; Ppi; M/A; UEVH-FUNGI 2003692.

\section{Amanita crocea (Quél.) Singer}

Material examined. Newly recorded (n). PORTUGAL

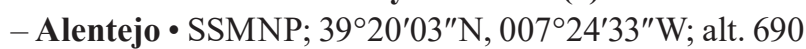
m; 14.XI.2016; Andrade J. leg.; M; Qp/Cs; Med.; UEVHFUNGI 2003609.

\section{Amanita curtipes E.-J. Gilbert}

Material examined. PORTUGAL - Alentejo - SSMNP; $39^{\circ} 10^{\prime} 11^{\prime \prime} \mathrm{N}, 007^{\circ} 09^{\prime} 37^{\prime \prime} \mathrm{W}$; alt. $350 \mathrm{~m}$; 30.XII.2015; Andrade J. leg.; M; Qs/Qr; Med.; UEVH-FUNGI 2003210.

Amanita franchetii (Boud.) Fayod (Fig. 4A)

Material examined. Newly recorded (n). PORTUGAL - Alentejo - SSMNP; 39 23'19"N, 007 $25^{\circ} 21^{\prime \prime} \mathrm{W}$; alt. 620m; 12.XI.2016; Andrade J. leg.; M; Qp/Cs; Med.; UEVH-FUNGI 2003592.

Identification. This species can be recognized by its conspicuous yellow colour. Pileus hemispheric to applanate, $4-8 \mathrm{~cm}$ wide, citrine yellow to sulphur yellow. Veil breaks up into small flat or pyramidal, yellowish scales. Stipe 1-2 cm long, tapering from a bulbous base girdled with yellowish scales. The membranous ring is grooved on its upper surface and has a yellow-brown edge. It can be distinguished from A. pantherina (DC.) Krombh., which has white veil remains, and from $A$. rubescens Pers. with grey to pink veil remains and a clear reaction (turning red) when cut or bruised.

\section{Amanita gemmata (Fr.) Bertill}

Figure 4B

Material examined. Newly recorded (n). PORTUGAL - Alentejo - SSMNP; 39 23'44"N, 007²5'09" W; alt. 580 m; 06.XII.2019; Andrade J. leg.; M; Qp/Qs; M/A; UEVH-FUNGI 2004794.

Identification. This species can be recognized by its hemispheric to applanate pileus, with darker depressed centre, yellow; growing to $10 \mathrm{~cm}$ in diameter; white veil fragments present mainly in centre. Hymenium white and crowded. Stipe up to $10 \mathrm{~cm}$ in height, white, swollen base, covered with a short volva. Usually has a very fragile and fleeting ring, below which has an arrow like pattern. It can be distinguished from Amanita citrina Pers., which is completely lime-coloured and has a distinctive potato-like smell.

\section{Amanita magnivolvata Aalto \\ Figure 4C}

Material examined. Newly recorded (n). PORTUGAL - Alentejo - SSMNP; 39²3'09"N, 007 21'42"W; alt. 640 m; 20.X.2019; Andrade J. leg.; M; Qp/Qs; M/A; UEVHFUNGI 2004752

Identification. This exceptionally large Amanita can be recognized by the oval-hemispherical to plano-convex pileus; smooth and shiny pileipellis, usually olive green, grey, or brownish, with a darker centre and a sulcate margin. Free, white to cream lamellae, with a white floccose margin. Stipe compact, white, cylindrical (sometimes wider at the base), striate at the apex and with no ring; surrounded by a fleshy, membranous, saccate broad volva $(10 \times 6 \mathrm{~cm}$ after drying) with yellowish spots. Can be distinguished from $A$. pachyvolvata (Bon) Krieglst. by its more oval ellipsoid spores.

Amanita muscaria (L.) Lam.

Material examined. PORTUGAL - Alentejo - SSMNP; $39^{\circ} 24^{\prime} 03^{\prime \prime} \mathrm{N}, 007^{\circ} 25^{\prime} 02^{\prime \prime} \mathrm{W}$; alt. $760 \mathrm{~m}$; 09.XI.2015; Andrade J. leg.; M; S; M/A; UEVH-FUNGI 2003105.

\section{Amanita pachyvolvata (Bon) Krieglst.}

Material examined. Newly recorded (n). PORTUGAL - Alentejo - SSMNP; $39^{\circ} 18^{\prime} 05^{\prime \prime} \mathrm{N}, 007^{\circ} 24^{\prime} 40^{\prime \prime} \mathrm{W}$; alt. 700 m; 12.XII.2017; Andrade J. leg.; M; Qp/Cs; M/A; UEVH-FUNGI 2003899.

\section{Amanita pantherina (DC.) Krombh.}

Material examined. PORTUGAL - Alentejo - SSMNP; $39^{\circ} 20^{\prime} 03^{\prime \prime} \mathrm{N}, 007^{\circ} 24^{\prime} 33^{\prime \prime} \mathrm{W}$; alt. $690 \mathrm{~m}$; 03.X.2016; Andrade J. leg.; M; Qp/Cs; M/A; UEVH-FUNGI 2003542.

\section{Amanita phalloides (Vaill. ex Fr.) Link}

Material examined. PORTUGAL - Alentejo - SSMNP; $39^{\circ} 19^{\prime} 15^{\prime \prime} \mathrm{N}, 007^{\circ} 24^{\prime} 47^{\prime \prime} \mathrm{W}$; alt. 600 m; 09.XI.2015; Andrade J. leg.; M; Qp/Qs; M/A; UEVH-FUNGI 2003080.

\section{Amanita rubescens Pers.}

Material examined. PORTUGAL - Alentejo - SSMNP; $39^{\circ} 18^{\prime} 03^{\prime \prime} \mathrm{N}, 007^{\circ} 24^{\prime} 43^{\prime \prime} \mathrm{W}$; alt. $680 \mathrm{~m}$; 10.XI.2015; Andrade J. leg.; M; Qp/Qs; M/A; UEVH-FUNGI 2003083.

\section{Amanita torrendii Justo}

Material examined. PORTUGAL - Alentejo - SSMNP; $39^{\circ} 29^{\prime} 45^{\prime \prime} \mathrm{N}, 007^{\circ} 27^{\prime} 03^{\prime \prime} \mathrm{W}$; alt. $370 \mathrm{~m}$; 01.V.2019; Andrade J. leg.; M; Qs/Qr; Med.; UEVH-FUNGI 2004740.

\section{Amanita vaginata (Bull.) Lam.}

Material examined. PORTUGAL - Alentejo - SSMNP; $39^{\circ} 23^{\prime} 09^{\prime \prime} \mathrm{N}, 007^{\circ} 21^{\prime} 42^{\prime \prime} \mathrm{W}$; alt. $640 \mathrm{~m}$; 08.XI.2016; Andrade J. leg.; M; Qp/Qs; M/A; UEVH-FUNGI 2003584.

Auriculariaceae

Auricularia auricula-judae (Bull.) Quél.

Material examined. PORTUGAL - Alentejo - SSMNP; $39^{\circ} 27^{\prime} 18^{\prime \prime} \mathrm{N}, 007^{\circ} 26^{\prime} 35^{\prime \prime} \mathrm{W}$; alt. $430 \mathrm{~m}$; 23.X.2013; Andrade J. leg.; S; Qs/Qr; M/A; UEVH-FUNGI 2002649.

\section{Bankeraceae}

\section{Hydnellum scrobiculatum (Fr.) P. Karst.} Figure 4D

Material examined. Newly recorded (n). PORTUGAL

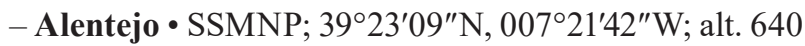




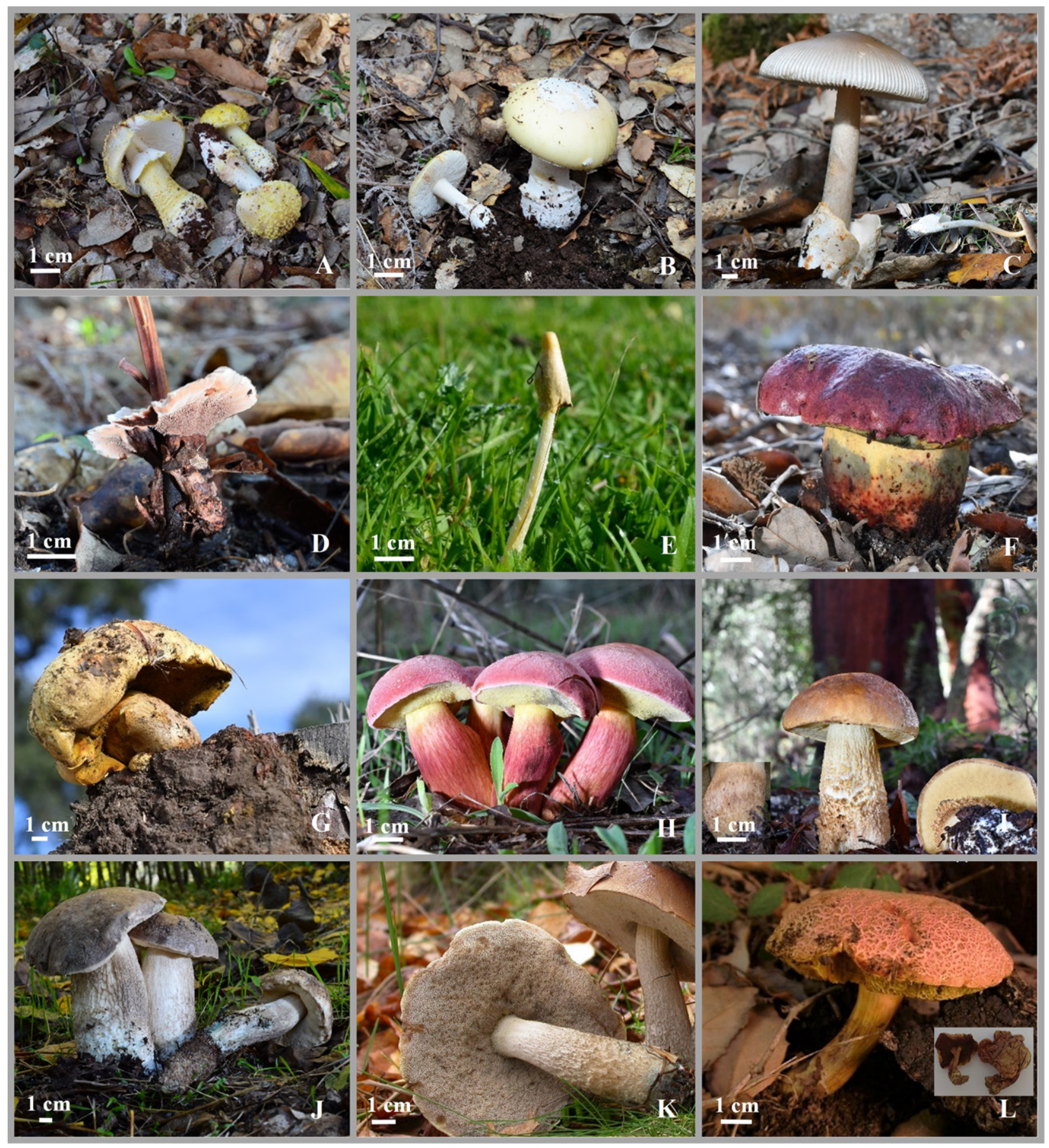

Figure 4. A. Amanita franchetii (Boud.) Fayod* B. Amanita gemmata (Fr.) Bertill C. Amanita magnivolvata Aalto** D. Hydnellum scrobiculatum (Fr.) P. Karst.* E. Bolbitius elegans E. Horak, G. Moreno, A. Ortega \& Esteve-Rav.** F. Boletus spretus Bertéa** G. Buchwaldoboletus hemichrysus (Berk. \& M.A. Curtis) Pilát** H. Hortiboletus rubellus (Krombh.) Simonini, Vizzini \& Gelardi* I. Leccinellum crocipodium (Letell.) Della Magg. \& Trassin.* J. Leccinum duriusculum (Schulzer ex Kalchbr.) Singer* K. Leccinum scabrum (Bull.) Gray* L. Rheubarbariboletus persicolor (H. Engel, Klofac, H. Grünert \& R. Grünert) Vizzini, Simonini \& Gelardi**. ( ${ }^{*}=$ newly recorded from Alto Alentejo Province; ${ }^{* *}=$ newly recorded from continental Portugal)

m; 04.XI.2019; Andrade J. leg.; M; Qp/Qs; M/A; UEVHFUNGI 2004769.

Identification. This species can be recognized by the applanate to infundibuliform pileus, up to $6 \mathrm{~cm}$ in diameter, concentrically zoned, ranging from dark rusty red in the centre to pinkish at the margin, velvety to smooth. Hymenium with purple spines up to $3 \mathrm{~mm}$ in length, running through the stipe. Cylindrical stipe, swollen at the base, velvety to spongy, concolourous with the pileus.
Spores irregularly ellipsoidal to subglobose, ornamented with irregular coarse warts. This species can be distinguished from $H$. concrescens (Pers.) Banker by its general reddish colouration.

\section{Phellodon niger (Fr.) P. Karst.}

Material examined. Newly recorded (n). PORTUGAL - Alentejo - SSMNP; 39¹8'06"N, 007²4'10"W; alt. 700 m; 02.XII.2016; Andrade J. leg.; S; Ppi; Med.; 


\section{UEVH-FUNGI 2003696.}

Bolbitiaceae

\author{
Bolbitius elegans E. Horak, G. Moreno, A. Ortega \& \\ Esteve-Rav. \\ Figure 4E
}

Material examined. Newly recorded (n). PORTUGAL - Alentejo - SSMNP; $39^{\circ} 23^{\prime} 44^{\prime \prime} \mathrm{N}, 007^{\circ} 25^{\prime} 09^{\prime \prime} \mathrm{W}$ alt. 580 m; 29.XI.2014; Andrade J. leg.; S; Qp/Qs; M/A; UEVHFUNGI 2002993.

Identification. This species can be recognized by its small, conical-campanulate pileus (never flattened after maturing) with a wavy, not striate margin; smooth, lemon-yellow pileipellis, viscid in wet weather, turning yellow-ferruginous or brown-orange-ochre upon maturity. Tight anastomosed lamellae, ochre-orange and deliquescent when aging. Tall lemon-yellow stipe, $4-11 \mathrm{~cm}$ long, thin, fistulous, and slightly pruinose. This species can be distinguished from B. titubans (Bull.) Fr. by microscopic features and by the applanate pileus upon maturity of B. titubans.

\section{Panaeolus rickenii Hora}

Material examined. Newly recorded (n). PORTUGAL - Alentejo • SSMNP; $39^{\circ} 18^{\prime} 03^{\prime \prime} \mathrm{N}, 007^{\circ} 24^{\prime} 43^{\prime \prime} \mathrm{W}$; alt. 680 m; 19.XI.2018; Andrade J. leg.; S; Qp/Qs; Att.; UEVHFUNGI 2004334.

\section{Boletaceae}

\section{Aureoboletus gentilis (Quél.) Pouzar}

Material examined. PORTUGAL - Alentejo • SSMNP; $39^{\circ} 23^{\prime} 09^{\prime \prime} \mathrm{N}, 007^{\circ} 21^{\prime} 42^{\prime \prime} \mathrm{W}$; alt. $640 \mathrm{~m}$; 11.XI.2018; Andrade J. leg.; M; Qp/Qs; M/A; UEVH-FUNGI 2004493.

\section{Boletus aereus Bull.}

Material examined. PORTUGAL - Alentejo • SSMNP; $39^{\circ} 29^{\prime} 45^{\prime \prime} \mathrm{N}, 007^{\circ} 27^{\prime} 03^{\prime \prime} \mathrm{W}$; alt. $370 \mathrm{~m}$; 06.XI.2019; Andrade J. leg.; M; Qs/Qr; Med.; UEVH-FUNGI 2004821.

\section{Boletus reticulatus Schaeff.}

Material examined. PORTUGAL - Alentejo • SSMNP; $39^{\circ} 08^{\prime} 04^{\prime \prime} \mathrm{N}, 007^{\circ} 12^{\prime} 37^{\prime \prime} \mathrm{W}$; alt. $370 \mathrm{~m}$; 15.V.2018; Andrade J. leg.; M; Qs/Qr; Med.; UEVH-FUNGI 2004132.

\section{Boletus spretus Bertéa}

Figure 4F

Material examined. Newly recorded (n). PORTUGAL - Alentejo • SSMNP; $39^{\circ} 19^{\prime} 17^{\prime \prime} \mathrm{N}, 007^{\circ} 24^{\prime} 54^{\prime \prime} \mathrm{W}$; alt. 600 m; 20.X.2019; Andrade J. leg.; M; Qp/Cs; Med.; UEVHFUNGI 2004756.

Identification. This species can be recognized by its often-clustered fruitbodies. Hemispherical, then planoconvex pileus, often with wavy margin, dry velvety pale red, red, or reddish-vinaceous pileipellis; becoming dark blue when bruised. Firm cylindrical to club-shaped stipe, cream or pale yellow to yellow; often reddish spotted in the lower part, covered with orange red, bright red, or vinaceous granules, blueing or blackening when bruised. Pale lemon flesh, blueing when exposed to air. Pale yellow tubes and pores, blueing or blackening when bruised. Boletus adonis Pöder \& H. Ladurner is very similar but has long tubes up to $17 \mathrm{~mm}$ in contrast to the short tubes (up to $5 \mathrm{~mm}$ ) typical for $B$. spretus.

\section{Boletus subtomentosus L.}

Material examined. PORTUGAL - Alentejo • SSMNP; $39^{\circ} 23^{\prime} 56^{\prime \prime} \mathrm{N}, 007^{\circ} 25^{\prime} 34^{\prime \prime} \mathrm{W}$; alt. $580 \mathrm{~m}$; 10.VI.2018; Andrade J. leg.; M; Qp/Qs; M/A; UEVH-FUNGI 2004156.

\section{Buchwaldoboletus hemichrysus (Berk. \& M.A. Curtis) Pilát \\ Figure 4G}

Material examined. Newly recorded (n). PORTUGAL - Alentejo • SSMNP; $39^{\circ} 19^{\prime} 56^{\prime \prime} \mathrm{N}, 007^{\circ} 22^{\prime} 52^{\prime \prime} \mathrm{W}$; alt. 710 m; 11.X.2019; Andrade J. leg.; S (on P. pinaster decaying wood); Ppi; Med.; UEVH-FUNGI 2004751.

Identification. This species can be recognized by its broadly convex, sturdy pileus with an exceeding margin, dry; bright yellow pileipellis, covered with fine yellow powder. Thick cylindrical stipe, very finely reticulate at the apex but smooth elsewhere, yellow toward apex and brownish red below. Yellow flesh in the stipe, whitish in the pileus; blueing when exposed to air, then turning pinkish. Dull yellow tubes and orange reddish pores, darker toward the margin, blueing when bruised. Boletus lignicola (Kallenb.) Pilát and B. sphaerocephalus (Barla) Watling \& T.H. Li are very similar species that do not have powdery pileipellis and differ in the pileus colour and habitat.

\section{Caloboletus radicans (Pers.) Vizzini}

Material examined. PORTUGAL - Alentejo - SSMNP; $39^{\circ} 19^{\prime} 15^{\prime \prime} \mathrm{N}, 007^{\circ} 24^{\prime} 47^{\prime \prime} \mathrm{W}$; alt. $600 \mathrm{~m}$; 06.XI.2016; Andrade J. leg.; M; Qp/Qs; Med.; UEVH-FUNGI 2003560.

\section{Hortiboletus rubellus (Krombh.) Simonini, Vizzini \& Gelardi \\ Figure $4 \mathrm{H}$}

Material examined. Newly recorded (n). PORTUGAL - Alentejo - SSMNP; $39^{\circ} 27^{\prime} 12^{\prime \prime} \mathrm{N}, 007^{\circ} 26^{\prime} 33^{\prime \prime} \mathrm{W}$; alt. 440 m; 04.XI.2019; Andrade J. leg.; M; Qp/Qs; Med.; UEVH-FUNGI 2004772.

Identification. This species can be recognized by the scarlet-red pileus, fading to olivaceous-red at maturity, convex to almost flat, dry, smooth, sometimes velvety, and rarely cracked. Margin paler than the pileus, usually yellowish. Tubes are yellow and terminate in lemon-yellow pores that become greenish with age, turning slowly blue when bruised. Stipe cylindrical, bright yellow at the apex, and progressively redder towards the base. Flesh dull yellow and turning blue when cut. Spores subfusiform to subellipsoidal, smooth, 11-12 $\times$ 5-6 $\mu \mathrm{m}$, olive-brown. This species can be distinguished from Hortiboletus engelii (Hlaváček) Biketova \& Wasser 
which has brown to pale brown pileus and from Rheubarbariboletus armeniacus due to the lack of vivid red colour towards the base of the stem in $R$. armeniacus.

\section{Lanmaoa fragrans (Vittad.) Vizzini, Gelardi \& Simonini}

Material examined. PORTUGAL - Alentejo - SSMNP; $39^{\circ} 23^{\prime} 56^{\prime \prime} \mathrm{N}, 007^{\circ} 25^{\prime} 34^{\prime \prime} \mathrm{W}$; alt. $580 \mathrm{~m}$; 15.X.2019; Andrade J. leg.; M; Qp/Qs; M/A; UEVH-FUNGI 2004749.

\section{Leccinellum crocipodium (Letell.) Della Magg. \& Trassin.}

Figure 4I

Material examined. Newly recorded (n). PORTUGAL - Alentejo - SSMNP; 39 $30^{\prime} 46^{\prime \prime} \mathrm{N}, 007^{\circ} 28^{\prime} 43^{\prime \prime} \mathrm{W}$; alt. 310 m; 10.VI.2018; Andrade J. leg.; M; S; Med.; UEVHFUNGI 2004157.

Identification. This species can be recognized by the hemispherical to convex pileus, reaching $14 \mathrm{~cm}$ in diameter, yellowish-brown or reddish-brown to dark ochre, strongly cracked at maturity. Hymenium composed of densely packed yellows tubes and lemon-yellow pores, darkening when bruised. Stipe cylindrical or bulbous towards the base, pale yellow, covered in woolly, brown scales, and reddening when bruised. Flesh yellowish, darkening when cut. This species can be distinguished from Leccinum duriusculum (Schulzer ex Kalchbr.) Singer by the strongly cracked pileus.

\section{Leccinellum lepidum (H. Bouchet ex Essette) Bresinsky \& Manfr. Binder}

Material examined. PORTUGAL - Alentejo - SSMNP; $39^{\circ} 23^{\prime} 09^{\prime \prime} \mathrm{N}, 007^{\circ} 21^{\prime} 42^{\prime \prime} \mathrm{W}$; alt. $640 \mathrm{~m}$; 31.X.2016; Andrade J. leg.; M; Qp/Qs; M/A; UEVH-FUNGI 2003566.

\section{Leccinum duriusculum (Schulzer ex Kalchbr.) Singer} Figure 4J

Material examined. Newly recorded (n). PORTUGAL - Alentejo - SSMNP; 39 $22^{\prime} 48^{\prime \prime} \mathrm{N}, 007^{\circ} 23^{\prime} 18^{\prime \prime} \mathrm{W}$; alt. 520 m; 05.XI.2016; Andrade J. leg.; M; Ppa; Atl.; UEVHFUNGI 2003551.

Identification. This species can be recognized by the hemispherical to convex pileus, up to $20 \mathrm{~cm}$ in diameter, ochre to greyish-brown, fibrillose-felted, finely cracked at maturity. Hymenium composed of pale grey tubes and densely packed pores, turning brown when bruised. Stipe cylindrical tapering at the apex, solid, pale grey, entirely covered with fine greyish to blackish squamules, which become larger towards the base, often arranged in longitudinal rows or ridges. Flesh turning yellow-green with blue spots on the base of the stipe. Spores fusiform with conical apex. This species differs from L. nigellum Redeuilh which has an almost black colour pileus.

\section{Leccinum scabrum (Bull.) Gray}

Figure 4K

Material examined. Newly recorded (n). PORTUGAL - Alentejo - SSMNP 39 $27^{\prime} 03^{\prime \prime} \mathrm{N}, 007^{\circ} 21^{\prime} 51^{\prime \prime} \mathrm{W}$; alt. 370 m; 06.XII.2019; Andrade J. leg.; M; Ppa; M/A; UEVHFUNGI 2004790.

Identification. This species can be recognized by the hemispherical to convex pileus, often deformed, up to 15 $\mathrm{cm}$ in diameter, grey-brown to brown with red tinges, initially finely tomentose but smooth at maturity. Hymenium composed by densely packed whitish tubes and pores, sometimes with brown spots, slowly turning brown when bruised. Stipe cylindrical or slightly bulbous at the base, pale yellow, covered in woolly brown scales, noticeably coarser at the base. Flesh white, turning light pink when exposed to air. Spores narrowly ellipsoid to subfusiform, with vacuolar inclusions. This species can be distinguished from L. cyaneobasileucum Lannoy \& Estadès and $L$. versipelle (Fr. \& Hök) Snell which turns to green or bluish at the base of the stipe when cut.

\section{Rheubarbariboletus armeniacus (Quél.) Vizzini, Simonini \& Gelardi}

Material examined. Newly recorded (n). PORTUGAL - Alentejo - SSMNP; 39 $18^{\prime} 15^{\prime \prime} \mathrm{N}, 007^{\circ} 24^{\prime} 35^{\prime \prime} \mathrm{W}$; alt. 720 m; 30.X.2016; Andrade J. leg.; M; Qp/Cs; Atl.; UEVHFUNGI 2003554.

Rheubarbariboletus persicolor (H. Engel, Klofac, H. Grünert \& R. Grünert) Vizzini, Simonini \& Gelardi Figure 4L

Material examined. Newly recorded (n). PORTUGAL - Alentejo - SSMNP; 39¹9'17"N, 007²4'54"W; alt. 600 m; 15.IV.2016; Andrade J. leg.; M; Qp/Cs; Med.; UEVHFUNGI 2003395.

Identification. This species can be recognized by its hemispherical then convex pileus, dry, velvety, and sometimes cracking, apricot to peach-coloured pileipellis. Cylindrical or spindle-shaped stipe, often curved at the base, pale yellow to yellow, gradually becoming orange to reddish orange at the lower half. Yellowish flesh in the cap, yellow in the stipe, orange yellow to apricot in the stipe base, blueing, mostly in the cap, when exposed to air. Lemon yellow tubes and pores, blueing when bruised. This species is very similar to R. armeniacus (Quél.) Vizzini, Simonini \& Gelardi from which it can be distinguished by the persisting apricot colour in the stipe base, even after drying.

\section{Suillellus comptus (Simonini) Vizzini, Simonini \& Gelardi}

Figure 5A

Material examined. Newly recorded (n). PORTUGAL - Alentejo • SSMNP; 39 27'12"N, 007 $26^{\prime} 33^{\prime \prime} \mathrm{W}$; alt. 440 m; 10.VI.2018; Andrade J. leg.; M; Qp/Qs; Med.; UEVHFUNGI 2004146.

Identification. This species can be recognized by its convex to plano-convex smooth pileus, greyish pink, flesh-coloured, pale pink, brownish or ochraceous pileipellis, usually spotted reddish brown in places, blueing when bruised. Cylindrical or club-shaped stipe, pale 


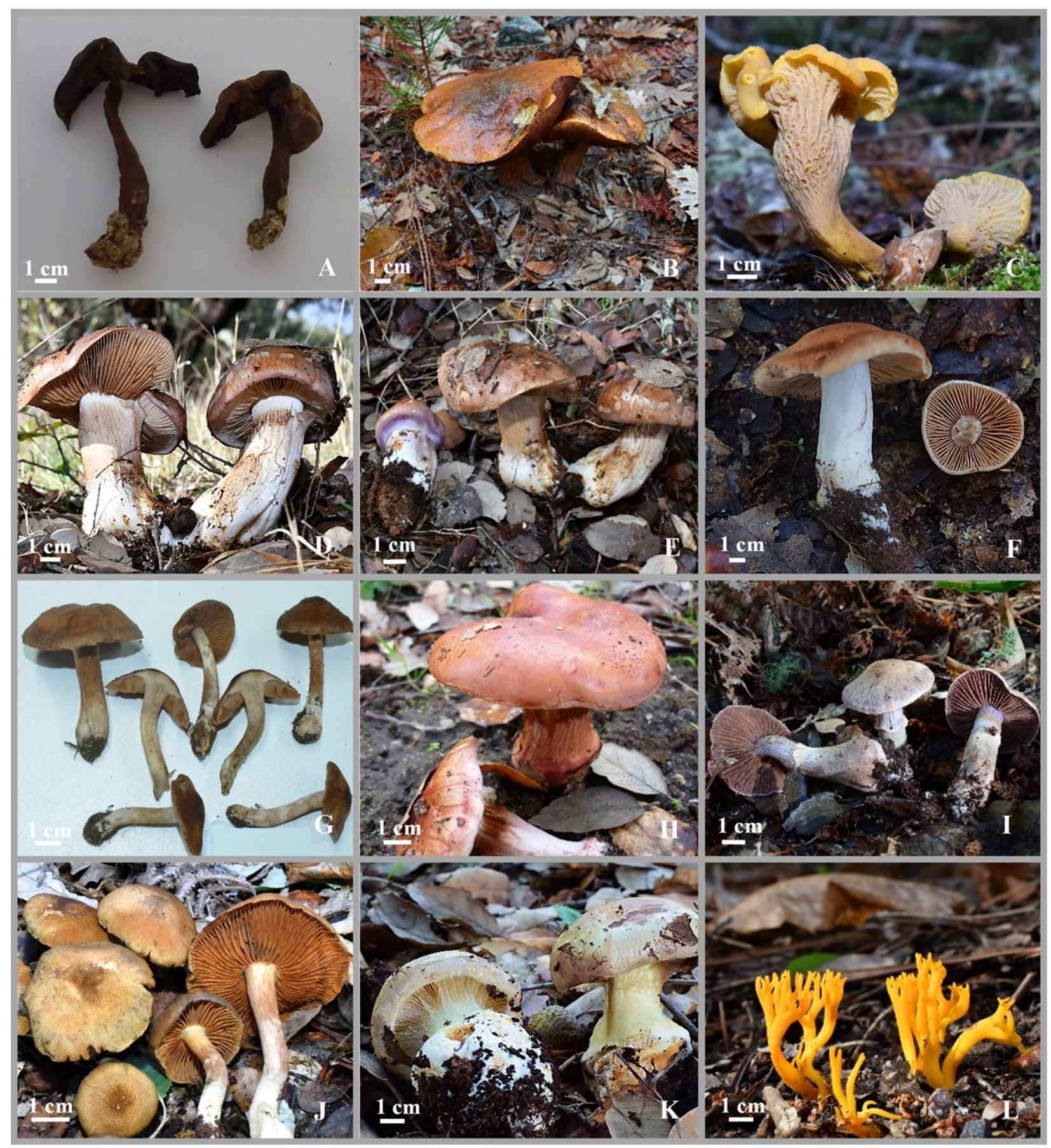

Figure 5. A. Suillellus comptus (Simonini) Vizzini, Simonini \& Gelardi** B. Suillellus queletii (Schulzer) Vizzini, Simonini \& Gelardi* C. Cantharellus melanoxeros Desm. ${ }^{* *}$ D. Cortinarius elegantior (Fr.) Fr. ${ }^{* *}$ E. Cortinarius largus Fr.* F. Cortinarius livido-ochraceus (Berk.) Berk. ${ }^{* *}$ G. Cortinarius rheubarbarinus Rob. Henry** H. Cortinarius rufo-olivaceus (Pers.) Fr.* I. Cortinarius torvus (Fr.) Fr.* J. Cortinarius venetus (Fr.) Fr.** K. Cortinarius xanthophyllus (Cooke) Rob. Henry** L. Calocera viscosa (Pers.) Fr... ${ }^{*}=$ newly recorded from Alto Alentejo Province; ${ }^{* *}=$ newly recorded from continental Portugal)

yellow to bright yellow, usually gradually darkening towards the base and becoming more reddish towards the apex; covered with yellow, orange, or pale red granules and blueing when bruised. Pale yellow to lemon-yellow flesh, spotted dark red to vinaceous red on the stipe base, blueing when exposed to air. Pale yellow to yellow tubes, blueing when injured; and red to orange-red pores, also blueing when bruised. Suillellus queletii (Schulzer) Vizzini, Simonini \& Gelardi is a similar species, which has a differently coloured pileus and a normally velvety rather than smooth surface.

\section{Suillellus luridus (Schaeff.) Murrill}

Material examined. Newly recorded (n). PORTUGAL - Alentejo • SSMNP; 39²7'12"N, 007²6'33"W; alt. 440 m; 11.X.2018; Andrade J. leg.; M; Qp/Qs; Med.; UEVHFUNGI 2004274.

\section{Suillellus queletii (Schulzer) Vizzini, Simonini \& Gelardi}

Figure 5B

Material examined. Newly recorded (n). PORTUGAL 
- Alentejo • SSMNP; 39²3'56"N, 007²5'34"W; alt. 580 m; 20.X.2019; Andrade J. leg.; M; Qp/Qs; M/A; UEVHFUNGI 2004775.

Identification. This species can be recognized by the broadly convex to applanate pileus, up to $18 \mathrm{~cm}$ in diameter, yellowish-orange to deep red, dry; pruinose at first but becoming smooth with age, bruising dark blue. Himenium composed of ochre tubes with apricot-orange pores that turn blue when handled. Stipe cylindrical or clavate, tapering at the base, yellow at the apex becoming increasingly redder towards the base, covered with fine granules, sometimes with a very faint reticule. Flesh yellow, becoming blue in the pileus when cut and red at the base of the stipe. Spores subfusiform to ellipsoidal, smooth. This species can be distinguished from S. luri$d u s$ (Schaeff.) Murrill by the absence of a distinct reticule on the stipe.

\section{Xerocomellus chrysenteron (Bull.) Šutara}

Material examined. PORTUGAL - Alentejo - SSMNP; $39^{\circ} 18^{\prime} 05^{\prime \prime} \mathrm{N}, 007^{\circ} 24^{\prime} 40^{\prime \prime} \mathrm{W}$; alt. 700 m; 28.X.2015; Andrade J. leg.; M; Qp/Cs; M/A; UEVH-FUNGI 2003225.

\section{Xerocomellus porosporus (Imler ex Watling) Šutara}

Material examined. Newly recorded (n). PORTUGAL - Alentejo • SSMNP; 39 $10^{\prime} 11^{\prime \prime} \mathrm{N}, 007^{\circ} 09^{\prime} 37^{\prime \prime} \mathrm{W}$; alt. 350 m; 15.X.2016; Andrade J. leg.; M; Qs/Qr; Med.; UEVHFUNGI 2003557.

\section{Xerocomus ferrugineus (Schaeff.) Alessio}

Material examined. PORTUGAL - Alentejo • SSMNP; $39^{\circ} 08^{\prime} 04^{\prime \prime} \mathrm{N}, 007^{\circ} 12^{\prime} 37^{\prime \prime} \mathrm{W}$; alt. $370 \mathrm{~m}$; 16.XII.2013; Andrade J. leg.; M; Qs/Qr; Med.; UEVH-FUNGI 2002683.

Cantharellaceae

\section{Cantharellus cibarius Fr.}

Material examined. PORTUGAL - Alentejo • SSMNP; $39^{\circ} 18^{\prime} 05^{\prime \prime} \mathrm{N}, 007^{\circ} 24^{\prime} 40^{\prime \prime} \mathrm{W}$; alt. $700 \mathrm{~m}$; 10.V.2018; Andrade J. leg.; M; Qp/Cs; M/A; UEVH-FUNGI 2004125.

\section{Cantharellus melanoxeros Desm.}

Figure 5C

Material examined. Newly recorded (n). PORTUGAL

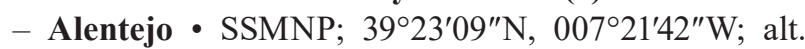
640 m; 06.XII.2019; Andrade J. leg.; M; Qp/Qs; M/A; UEVH-FUNGI 2004781.

Identification. This species can be recognized by its applanate to wavy-sinuous pileus, often with a rolled-up margin. Saffron-yellow pileipellis with lilac tints, blackening with age. Rose lilac-purple hymenium with decurrent, highly anastomosed or irregularly wavy folds. Stipe cylindrical or slightly tapered downwards, smooth, whitish turning to pink-lilac. Flesh purplish or blackened on the base of the stipe. Cantharellus amethysteus (Quél.) Sacc. has an orange or amethyst-lilac pileipellis and yellow hymenium, and $C$. ianthinoxanthus (Maire) Kühner does not blacken, and its spores are larger.

\section{Cantharellus pallens Pilát}

Material examined. PORTUGAL - Alentejo - SSMNP; $39^{\circ} 18^{\prime} 05^{\prime \prime} \mathrm{N}, 007^{\circ} 24^{\prime} 40^{\prime \prime} \mathrm{W}$; alt. $700 \mathrm{~m}$; 11.XII.2016; Andrade J. leg.; M; Qp/Qs; M/A; UEVH-FUNGI 2003739.

\section{Clavulina cinerea (Bull.) J. Schröt.}

Material examined. PORTUGAL - Alentejo • SSMNP; $39^{\circ} 18^{\prime} 05^{\prime \prime} \mathrm{N}, 007^{\circ} 24^{\prime} 40^{\prime \prime} \mathrm{W}$; alt. 700 m; 30.XI.2015; Andrade J. leg.; M; Qp/Cs; M/A; UEVH-FUNGI 2004904.

\section{Clavulina coralloides (L.) J. Schröt.}

Material examined. PORTUGAL - Alentejo - SSMNP; $39^{\circ} 23^{\prime} 09^{\prime \prime} \mathrm{N}, 007^{\circ} 21^{\prime} 42^{\prime \prime} \mathrm{W}$; alt. 640 m; 27.XI.2016; Andrade J. leg.; M; Qp/Qs; M/A; UEVH-FUNGI 2003667.

\section{Clavulina rugosa (Bull.) J. Schröt.}

Material examined. PORTUGAL - Alentejo - SSMNP; $39^{\circ} 24^{\prime} 23^{\prime \prime} \mathrm{N}, 007^{\circ} 24^{\prime} 31^{\prime \prime} \mathrm{W}$; alt. $660 \mathrm{~m}$; 22.X.2013; Andrade J. leg.; M; Qp/Cs; M/A; UEVH-FUNGI 2002266.

\section{Craterellus cornucopioides (L.) Pers.}

Material examined. PORTUGAL - Alentejo - SSMNP; $39^{\circ} 18^{\prime} 15^{\prime \prime} \mathrm{N}, 007^{\circ} 24^{\prime} 35^{\prime \prime} \mathrm{W}$; alt. $720 \mathrm{~m}$; 18.II.2020; Andrade J. leg.; M; Qp/Cs; Atl.; UEVH-FUNGI 2004898.

\section{Craterellus tubaeformis (Fr.) Quél.}

Material examined. PORTUGAL - Alentejo - SSMNP; $39^{\circ} 22^{\prime} 28^{\prime \prime} \mathrm{N}, 007^{\circ} 22^{\prime} 08^{\prime \prime} \mathrm{W}$; alt. $720 \mathrm{~m}$; 06.I.2018; Andrade J. leg.; M; Ppi; M/A; UEVH-FUNGI 2003915.

\section{Cortinareaceae}

\section{Cortinarius balteatocumatilis Rob. Henry ex P.D. Orton}

Material examined. Newly recorded (n). PORTUGAL - Alentejo • SSMNP; 39 23'09"N, 007²1'42"W; alt. 640 m; 19.XI.2018; Andrade J. leg.; M; Qp/Qs; M/A; UEVHFUNGI 2004321.

\section{Cortinarius croceus (Schaeff.) Gray}

Material examined. Newly recorded (n). PORTUGAL - Alentejo • SSMNP; 39 $19^{\prime} 56^{\prime \prime} \mathrm{N}, 007^{\circ} 22^{\prime} 52^{\prime \prime} \mathrm{W}$; alt. 710 m; 11.XII.2016; Andrade J. leg.; M; Ppi; Alt.; UEVHFUNGI 2003713.

\section{Cortinarius elegantior (Fr.) Fr.}

Figure 5D

Material examined. Newly recorded (n). PORTUGAL - Alentejo - SSMNP; 39 $18^{\prime} 03^{\prime \prime} \mathrm{N}, 007^{\circ} 24^{\prime} 43^{\prime \prime} \mathrm{W}$; alt. 680 m; 16.XI.2018; Andrade J. leg.; M; Qp/Qs; Alt.; UEVHFUNGI 2004318.

Identification. This species can be recognized by its large size, reaching $15 \mathrm{~cm}$ in diameter. Pileus yellowochre, ochre-tawny, with reddish-brown spots; yellowish lamellae turning purple-brown or rust-coloured when mature. Yellowish stipe with marginate bulb wrapped in a brown-yellowish veil. Yeast or malt smell and taste; slightly pink reaction to $\mathrm{KOH}$ at the base of the bulb. 
Agmidaliform to citriform warty spores, $13 \times 8 \mu \mathrm{m}$. A similar species, C. quercus-ilicis (Chevassut \& Rob. Henry) Melot, can be distinguished for its occurrence in more xerophytic environments and smaller spore size.

\section{Cortinarius glaucopus (Schaeff.) Gray}

Material examined. Newly recorded (n). PORTUGAL

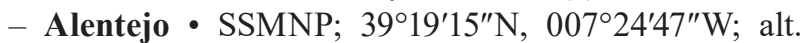
600 m; 06.XII.2019; Andrade J. leg.; M; Qp/Qs; M/A; UEVH-FUNGI 2004791.

\section{Cortinarius infractus (Pers.) Fr.}

Material examined. Newly recorded (n). PORTUGAL - Alentejo • SSMNP; 39 $19^{\prime} 15^{\prime \prime} \mathrm{N}, 007^{\circ} 24^{\prime} 47^{\prime \prime} \mathrm{W}$; alt. 600 m; 16.XI.2018; Andrade J. leg.; M; Qp/Qs; M/A; UEVHFUNGI 2004387.

\section{Cortinarius largus Fr.}

Figure 5E

Material examined. Newly recorded (n). PORTUGAL - Alentejo - SSMNP; 39²4'23"N, 007²4'31" W; alt. 660 m; 03.XII.2016; Andrade J. leg.; M; Qp/Cs; M/A; UEVH-FUNGI 2003698.

Identification. This species can be recognized by its hemispheric to applanate pileus, 3-12 cm wide, grey-lilac to brownish purple, darkening brown from the centre. Curved margin with veil remnants. Hymenium initially purplish, becoming dark brown with age. Stipe ranging between 5-10 $\mathrm{cm}$ in length, club-shaped, purplish and swollen at the base. Flesh violet especially near the base of the stipe. Faint smell and mild taste. Agmidaliform to citriform warty spores measuring 9.5-11 × 5-7 $\mu \mathrm{m}$. A similar species, Cortinarius variicolor (Pers.) Fr., can be distinguished by its earthy odour and darker radial fibrils on the pileus.

\section{Cortinarius livido-ochraceus (Berk.) Berk.}

Figure 5F

Material examined. Newly recorded (n). PORTUGAL - Alentejo - SSMNP; 39 $24^{\prime} 23^{\prime \prime} \mathrm{N}, 007^{\circ} 24^{\prime} 31^{\prime \prime} \mathrm{W}$; alt. 660 m; 03.XII.2016; Andrade J. leg.; M; Qp/Cs; M/A; UEVH-FUNGI 2003698.

Identification. This species can be recognized by its shiny, viscous, yellowish brown or olive-ochre pileus, with its characteristic wavy or wrinkled margin. Beige anastomosed lamellae, turning brown upon maturity, typically with crenelated whitish edges. Stipe viscous, cylindrical or tapering towards the base, whitish to purplish (veil), often striate at the top. Smell strong of honey. This species can be distinguished from C. pseudosalor J.E. Lange by its wavy or wrinkled margin, and from $C$. mucifluus Fr., which is also very similar, but has a white stipe with no striations on top.

\section{Cortinarius rheubarbarinus Rob. Henry} Figure $5 \mathrm{G}$

Material examined. Newly recorded (n). PORTUGAL - Alentejo • SSMNP; 39²3'44"N, 007²5'09" W; alt. 580 m; 26.XI.2019; Andrade J. leg.; M; Qp/Qs; M/A; UEVHFUNGI 2004803.

Identification. This species can be recognized by its convex to umbonate pileus, strongly hygrophane, brown at the umbo and lighter towards the margin and ochreemarginated lamellae. Fusoid stipe with a slight claviform base, often greyish white, silvery, or bluish at the top. The strong smell of parsley or nutmeg is its most discriminating feature. Although both C. craticius Fr. and $C$. pholideus (Lilj.) Fr. have a similar odour, they are morphologically very different from $C$. rheubarbarinus.

\section{Cortinarius rufo-olivaceus (Pers.) Fr.}

Figure $5 \mathrm{H}$

Material examined. Newly recorded (n). PORTUGAL - Alentejo - SSMNP; 39 $23^{\prime} 44^{\prime \prime} \mathrm{N}, 007^{\circ} 25^{\prime} 09^{\prime \prime} \mathrm{W}$; alt. 580 m; 22.XII.2014; Andrade J. leg.; M; Qp/Qs; M/A; UEVH-FUNGI 2002928.

Identification. Hemispherical to convex or plano-convex pileus, up to $10 \mathrm{~cm}$ in diameter, sometimes depressed, pink- to purple-red or reddish-copper, with paler margin. Stipe cylindrical, pale lilaceous, emarginated by a winered veil, bulbous base with purple-brown to wine-brown tinges. Lamellae broadly attached, greenish to lemonyellow when young, then dark rusty with age. Lemonshaped spores, $11-13 \times 6-7.5 \mu \mathrm{m}$ and short, clavate basidia, 40-45 × 10-12 $\mu \mathrm{m}$. It can be distinguished from Cortinarius prasinus (Schaeff.) Fr., which has smaller spores and basidia, and from the C. cupreorufus Brandrud, which inhabits mostly coniferous forests.

\section{Cortinarius torvus (Fr.) Fr.}

Figure 5I

Material examined. Newly recorded (n). PORTUGAL - Alentejo - SSMNP; 39²3'09"N, 007 21'42"W; alt. 640 m; 16.XI.2018; Andrade J. leg.; M; Qp/Qs; M/A; UEVHFUNGI 2004284.

Identification. This species can be recognized by the convex to broadly convex pileus, up to $10 \mathrm{~cm}$ across, covered with pallid radial fibrils, lilac-brown when young, fading as it dries to greyish lilac. Hymenium brownish purple when young, becoming rusty brown, covered by a whitish veil when young. Stipe club-shaped, clavate, $4-10 \mathrm{~cm}$ in length, thicker at the apex, pale purple; sheathed from the base with whitish to lilac-grey veil which often terminates in a folded-over, fragile ring. Spores ovoid and strongly verrucose. Cortinarius anomalus (Fr.) Fr. inhabits the same habitats but does not have the lower half of the stipe covered in white veil. It may also be confused with $C$. mucifluoides Rob. Henry ex Bidaud, Moënne-Locc. \& Reumaux which does not have a clavate stipe, and the lower half of the stipe is covered in a purplish, rather than white veil.

\section{Cortinarius trivialis J.E. Lange}

Material examined. PORTUGAL - Alentejo - SSMNP; $39^{\circ} 19^{\prime} 15^{\prime \prime} \mathrm{N}, \quad 007^{\circ} 24^{\prime} 47^{\prime \prime} \mathrm{W}$; alt. $600 \mathrm{~m}$; 09.XI.2015; 
Andrade J. leg.; M; Qp/Qs; M/A; UEVH-FUNGI 2003079.

\section{Cortinarius venetus ( $\mathrm{Fr}$.) Fr.}

Figure 5J

Material examined. Newly recorded (n). PORTUGAL - Alentejo • SSMNP; 39 $23^{\prime} 09^{\prime \prime} \mathrm{N}, 007^{\circ} 21^{\prime} 42^{\prime \prime} \mathrm{W}$; alt. 640 m; 04.XI.2019; Andrade J. leg.; M; Qp/Qs; M/A; UEVHFUNGI 2004766.

Identification. This species can be recognized by its velvety-scaly, yellow-olive, convex to plano-convex (often with a wide umbo) hygrophane pileus. Thick, separated lamellae, yellow-olive, turning ochre-ferruginous after spore maturation. Firm, cylindrical, yellowish-olive stipe, slightly bulbous towards the base, with a fleeting yellow veil. Strong radish smell. The similar $C$. cotoneus Fr. can be distinguished by its larger size.

\section{Cortinarius xanthophyllus (Cooke) Rob. Henry} Figure 5K

Material examined. Newly recorded (n). PORTUGAL

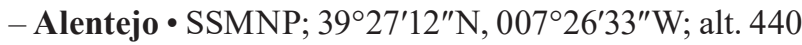
m; 16.XI.2018; Andrade J. leg.; M; Qp/Qs; Med.; UEVHFUNGI 2004328.

Identification. This species can be recognized by its hemispherical to convex pileus with rolled margin, colour ranging from purple-lilac or blue, then greenish grey to olive brown, viscous in wet weather. Citrus-yellow lamellae. Greenish-yellow stipe with marginate bulb. Whitish flesh on the pileus, yellowish on the stipe, and pinkish on the bulb. KOH reaction red dark on pileipellis, and yellow-green becoming red-purple on the inner flesh. This species can be distinguished from the similar C. splendens Rob. Henry by the pileus color, which is sulphur yellow in C. splendens.

Dacrymycetaceae

\section{Calocera cornea (Batsch) Fr.}

Material examined. PORTUGAL - Alentejo • SSMNP; $39^{\circ} 23^{\prime} 09^{\prime \prime} \mathrm{N}, 007^{\circ} 21^{\prime} 42^{\prime \prime} \mathrm{W}$; alt. $640 \mathrm{~m}$; 19.II.2020; Andrade J. leg.; S; Qp/Qs; M/A; UEVH-FUNGI 2004906.

\section{Calocera viscosa (Pers.) Fr.}

Figure 5L

Material examined. Newly recorded (n). PORTUGAL - Alentejo • SSMNP; $39^{\circ} 18^{\prime} 15^{\prime \prime} \mathrm{N}, 007^{\circ} 24^{\prime} 35^{\prime \prime} \mathrm{W}$; alt. 720 m; 19.II.2020; Andrade J. leg.; S; Qp/Cs; M/A; UEVHFUNGI 2004905.

Identification. This species can be recognized by the bright orange basidiocarps, up to $10 \mathrm{~cm}$ in length, very greasy and viscid, composed by several antlerlike branches forked at the tip. In dry weather it can become red-orange. Spores ellipsoidal, $8-12 \times 3.5-5 \mu \mathrm{m}$, hyaline, inamyloid; sometimes becoming septate when fully mature, each spore containing two oil droplets. This species can easily be distinguished from Ramaria Fr. ex Bonord. spp. by the very greasy and viscid surface.
Diplocystidiaceae

\section{Astraeus hygrometricus (Pers.) Morgan}

Material examined. PORTUGAL - Alentejo • SSMNP; $39^{\circ} 27^{\prime} 12^{\prime \prime} \mathrm{N}, 007^{\circ} 26^{\prime} 33^{\prime \prime} \mathrm{W}$; alt. $440 \mathrm{~m}$; 05.IV.2017; Andrade J. leg.; M; Qp/Qs; Med.; UEVH-FUNGI 2004023.

\section{Entolomataceae}

Clitopilus cystidiatus Hauskn. \& Noordel.

Material examined. Newly recorded (n). PORTUGAL - Alentejo • SSMNP; $39^{\circ} 19^{\prime} 15^{\prime \prime} \mathrm{N}, 007^{\circ} 24^{\prime} 47^{\prime \prime} \mathrm{W}$; alt. 600 m; 30.X.2016; Andrade J. leg.; S; Qp/Qs; M/A; UEVHFUNGI 2003568.

Entoloma clypeatum (L.) P. Kumm.

Material examined. PORTUGAL - Alentejo • SSMNP; $39^{\circ} 24^{\prime} 23^{\prime \prime} \mathrm{N}, 007^{\circ} 24^{\prime} 31^{\prime \prime} \mathrm{W}$; alt. $660 \mathrm{~m}$; 17.XI.2015; Andrade J. leg.; M; Qp/Cs; M/A; UEVH-FUNGI 2003144.

\section{Entoloma hirtipes (Schumach.) M.M. Moser \\ Figure 6A}

Material examined. Newly recorded (n). PORTUGAL - Alentejo • SSMNP; $39^{\circ} 08^{\prime} 15^{\prime \prime} \mathrm{N}, 007^{\circ} 12^{\prime} 54^{\prime \prime} \mathrm{W}$; alt. 370 m; 11.XI.2018; Andrade J. leg.; M; Qs/Qr; Med.; UEVHFUNGI 2004487.

Identification. This species can be recognized by the conical pileus, with a very prominent centre; hygrophanous, ranging from dark brown when wet to grey when dry, with radial fibrils. Crowded lamellae, slightly paler than the pileus, with a pinkish tone. Stipe slender, fistulous, the same colour as the pileus; mycelial cords present on the base. Spores size 10-14 $\times 8-9 \mu \mathrm{m}$, with 5-7 angles, yellowish. It can be confused with Entoloma hebes (Romagn.) Trimbach, which has smaller spores, only to $10 \mu \mathrm{m}$ in length, and occurs mostly in autumn, in contrast to E. hirtipes which occurs during spring.

\section{Entoloma rhodopolium (Fr.) P. Kumm.}

Material examined. PORTUGAL - Alentejo • SSMNP; $39^{\circ} 19^{\prime} 15^{\prime \prime} \mathrm{N}, 007^{\circ} 24^{\prime} 47^{\prime \prime} \mathrm{W}$; alt. $600 \mathrm{~m}$; 24.XI.2016; Andrade J. leg.; M; Qp/Qs; M/A; UEVH-FUNGI 2003628.

\section{Entoloma sinuatum (Bull.) P. Kumm.}

Material examined. PORTUGAL - Alentejo • SSMNP; $39^{\circ} 23^{\prime} 09^{\prime \prime} \mathrm{N}, 007^{\circ} 21^{\prime} 42^{\prime \prime} \mathrm{W}$; alt. $640 \mathrm{~m}$; 11.XI.2018; Andrade J. leg.; M; Qp/Qs; M/A; UEVH-FUNGI 2004299.

Fistulinaceae

\section{Fistulina hepatica (Schaeff.) With.}

Figure 6B

Material examined. Newly recorded (n). PORTUGAL - Alentejo • SSMNP; $39^{\circ} 23^{\prime} 19^{\prime \prime} \mathrm{N}, 007^{\circ} 25^{\prime} 21^{\prime \prime} \mathrm{W}$; alt. 620 m; 09.XI.2015; Andrade J. leg.; S; Qp/Cs; M/A; UEVHFUNGI 2003103.

Identification. This species can be recognized by the irregularly shaped pileus, up to $25 \mathrm{~cm}$ in diameter, often semi-circular, fan-shaped, or tongue-like, with a lobed, 


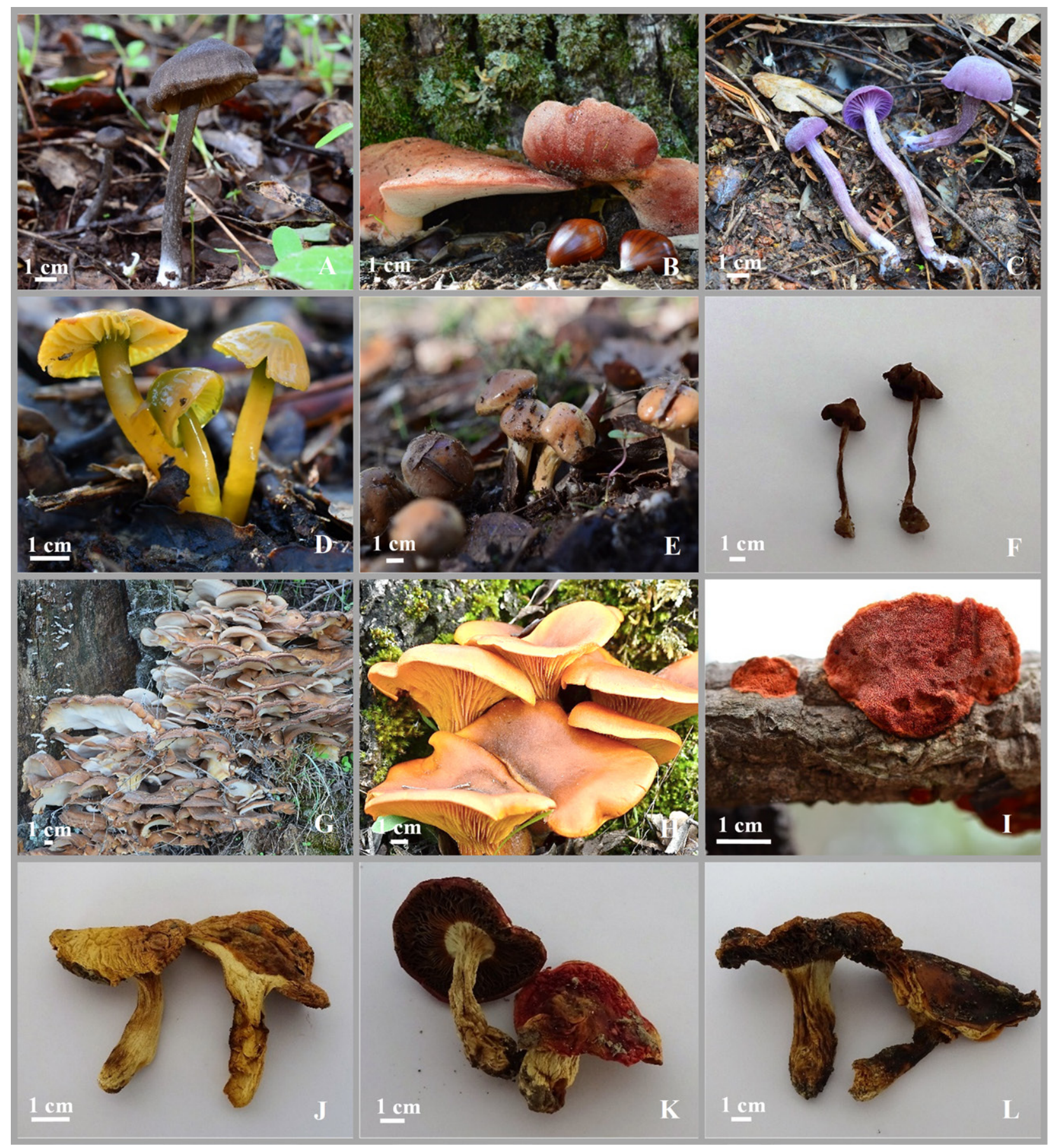

Figure 6. A. Entoloma hirtipes (Schumach.) M.M. Moser* B. Fistulina hepatica (Schaeff.) With. ${ }^{*}$ C. Laccaria amethystina Cooke* D. Gliophorus psittacinus (Schaeff.) Herink* E. Hebeloma collariatum Bruchet** F. Inocybe acuta Boud. ${ }^{* *}$ G. Meripilus giganteus (Pers.) P. Karst.* H. Omphalotus illudens (Schwein.) Bresinsky \& BesI ${ }^{* *}$ I. Pycnoporus cinnabarinus (Jacq.) P. Karst..* J. Russula farinipes Romell ${ }^{* *}$ K. Russula oreades Sarnari** L. Russula sericatula Romagn. ${ }^{* *} .{ }^{*}=$ newly recorded from Alto Alentejo Province; ${ }^{* *}=$ newly recorded from continental Portugal)

wavy margin; smooth, hygrophanous, viscous when wet, ranging from dark orange to dark red. Hymenium composed of crowded, yellowish pores, bruising cherry red. Stipe rarely present. Inonotus hispidus (Bull.) P. Karst. is similar but has a hairy or velvety upper surface and occurs in different habitats.

Geastraceae

\section{Geastrum campestre Morgan}

Material examined. PORTUGAL - Alentejo • SSMNP; $39^{\circ} 23^{\prime} 09^{\prime \prime} \mathrm{N}, 007^{\circ} 21^{\prime} 42^{\prime \prime} \mathrm{W}$; alt. $640 \mathrm{~m}$; 03.XII.2019;
Andrade J. leg.; S; Qp/Qs; M/A; UEVH-FUNGI 2004776.

Gomphidiaceae

\section{Chroogomphus fulmineus (R. Heim) Courtec.}

Material examined. PORTUGAL - Alentejo - SSMNP; $39^{\circ} 18^{\prime} 06^{\prime \prime} \mathrm{N}, 007^{\circ} 24^{\prime} 10^{\prime \prime} \mathrm{W}$; alt. 700 m; 05.XII.2016; Andrade J. leg.; M; Ppi; M/A; UEVH-FUNGI 2003694.

\section{Chroogomphus rutilus (Schaeff.) O.K. Mill.}

Material examined. Newly recorded (n). PORTUGAL - Alentejo - SSMNP; 39 $19^{\prime} 17^{\prime \prime} \mathrm{N}, 007^{\circ} 23^{\prime} 08^{\prime \prime} \mathrm{W}$; alt. 
770 m; 03.I.2019; Andrade J. leg.; M; Ppi; M/A; UEVHFUNGI 2004707.

Gyroporaceae

Gyroporus castaneus (Bull.) Quél.

Material examined. PORTUGAL - Alentejo - SSMNP; $39^{\circ} 28^{\prime} 11^{\prime \prime} \mathrm{N}, 007^{\circ} 25^{\prime} 04^{\prime \prime}$ Walt. 400 m; 10.X.2014; Andrade J. leg.; M; Qs/Qr; Med.; UEVH-FUNGI 2002960.

Hydnaceae

\section{Hydnum repandum $\mathrm{L}$.}

Material examined. PORTUGAL - Alentejo - SSMNP; $39^{\circ} 18^{\prime} 05^{\prime \prime} \mathrm{N}, 007^{\circ} 24^{\prime} 40^{\prime \prime} \mathrm{W}$; alt. $700 \mathrm{~m}$; 19.I.2018; Andrade J. leg.; M; Qp/Cs; M/A; UEVH-FUNGI 2003961.

Hydnangiaceae

\section{Laccaria amethystina Cooke}

Figure 6C

Material examined. Newly recorded (n). PORTUGAL - Alentejo - SSMNP; $39^{\circ} 18^{\prime} 05^{\prime \prime} \mathrm{N}, 007^{\circ} 24^{\prime} 40^{\prime \prime} \mathrm{W}$; alt. 700 m; 03.XII.2016; Andrade J. leg.; M; Qp/Cs; M/A; UEVH-FUNGI 2003672.

Identification. This species can be recognized by the concolourous pileus, hymenium, and stipe. Pileus 2-7 $\mathrm{cm}$ in diameter, deep or dark purple when moist and light purple to lilac when dry, pruinose, depressed at the centre and striate at the margin. Margin slightly curved. Hymenium composed of broad dark purple lamellae, widely spaced and with intermediate lamellulae. Stipe clavate, twisted radially, fibrillose, deep purple, mycelial cords present. Spores spherical covered with spines. This species can be distinguished from Mycena pura (Pers.) P. Kumm. which tends to be much paler than L. amethystina and has pale pink lamellae.

\section{Laccaria laccata (Scop.) Cooke}

Material examined. PORTUGAL - Alentejo - SSMNP; $39^{\circ} 23^{\prime} 44^{\prime \prime} \mathrm{N}, 007^{\circ} 25^{\prime} 09^{\prime \prime} \mathrm{W}$; alt. 580 m; 24.XI.2015; Andrade J. leg.; M; Qp/Qs; M/A; UEVH-FUNGI 2003046.

Hygrophoraceae

\section{Cuphophyllus russocoriaceus (Berk. \& T.K. Mill.) Bon}

Material examined. PORTUGAL - Alentejo • SSMNP; $39^{\circ} 08^{\prime} 15^{\prime \prime} \mathrm{N}, 007^{\circ} 12^{\prime} 54^{\prime \prime} \mathrm{W}$; alt. $370 \mathrm{~m}$; 09.XII.2015; Andrade J. leg.; S; Qs/Qr; Med.; UEVH-FUNGI 2003176.

\section{Gliophorus psittacinus (Schaeff.) Herink}

Figure 6D

Material examined. Newly recorded (n). PORTUGAL

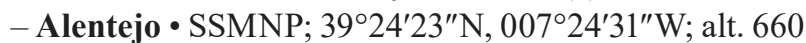
m; 12.III.2020; Andrade J. leg.; S; Qp/Cs; M/A; UEVHFUNGI 2004901

Identification. This species can be recognized by the green pileus and stipe. Pileus bell-shaped, up to $4 \mathrm{~cm}$ in diameter, becoming umbonate with age; initially green, soon turning yellowish-green, viscid when wet. Margin striate. Lamellae thick, waxy, overall cream-coloured with greenish or yellowish hues. Stipe solid, straight, and green near the apex, becoming yellow near the base. Spores smooth and ellipsoid. This species can be distinguished from Hygrocybe chlorophana (Fr.) Wünsche which exhibits a yellow pileus when young and a hollow stipe at maturity.

\section{Hygrocybe acutoconica (Clem.) Singer}

Material examined. Newly recorded (n). PORTUGAL - Alentejo • SSMNP; 39 $29^{\prime} 45^{\prime \prime} \mathrm{N}, 007^{\circ} 27^{\prime} 03^{\prime \prime} \mathrm{W}$; alt. 370 m; 10.V.2018; Andrade J. leg.; S; Qs/Qr; Med.; UEVHFUNGI 2004177.

\section{Hygrocybe conica (Schaeff.) P. Kumm.}

Material examined. PORTUGAL - Alentejo - SSMNP; $39^{\circ} 18^{\prime} 03^{\prime \prime} \mathrm{N}, 007^{\circ} 24^{\prime} 43^{\prime \prime} \mathrm{W}$; alt. 680 m; 12.XI.2016; Andrade J. leg.; S; Qp/Qs; M/A; UEVH-FUNGI 2003604.

\section{Hygrophorus cossus (Sowerby) Fr.}

Material examined. PORTUGAL - Alentejo - SSMNP; $39^{\circ} 30^{\prime} 46^{\prime \prime} \mathrm{N}, 007^{\circ} 28^{\prime} 43^{\prime \prime} \mathrm{W}$; alt. 310 m; 05.XI.2016; Andrade J. leg.; M; S; Med.; UEVH-FUNGI 2003555.

\section{Hygrophorus persoonii Arnolds}

Material examined. PORTUGAL - Alentejo - SSMNP; $39^{\circ} 23^{\prime} 44^{\prime \prime} \mathrm{N}, 007^{\circ} 25^{\prime} 09^{\prime \prime} \mathrm{W}$; alt. $580 \mathrm{~m}$; 11.XII.2016; Andrade J. leg.; M; Qp/Qs; M/A; UEVH-FUNGI 2003738.

Hygrophoropsidaceae

\section{Hygrophoropsis aurantiaca (Wulfen) Maire}

Material examined. PORTUGAL - Alentejo - SSMNP; $39^{\circ} 17^{\prime} 10^{\prime \prime} \mathrm{N}, 007^{\circ} 20^{\prime} 57^{\prime \prime} \mathrm{W}$; alt. $770 \mathrm{~m}$; 05.XII.2016; Andrade J. leg.; S; Ppi; M/A; UEVH-FUNGI 2003704.

Hymenochaetaceae

\section{Coltricia perennis (L.) Murrill}

Material examined. PORTUGAL - Alentejo - SSMNP; $39^{\circ} 18^{\prime} 15^{\prime \prime} \mathrm{N}, 007^{\circ} 24^{\prime} 35^{\prime \prime} \mathrm{W}$; alt. $720 \mathrm{~m}$; 14.III.2020; Andrade J. leg.; M; Qp/Cs; Atl.; UEVH-FUNGI 2004866.

Hymenogastraceae

\section{Gymnopilus junonius (Fr.) P. D. Orton}

Material examined. PORTUGAL - Alentejo - SSMNP; $39^{\circ} 23^{\prime} 44^{\prime \prime} \mathrm{N}, 007^{\circ} 25^{\prime} 09^{\prime \prime} \mathrm{W}$; alt. 580 m; 06.XI.2016; Andrade J. leg.; $\mathbf{S}$ (on Q. pyrenaica stumps and trunks); Qp/ Qs; M/A; UEVH-FUNGI 2003571.

\section{Gymnopilus suberis (Maire) Singer}

Material examined. PORTUGAL - Alentejo - SSMNP; $39^{\circ} 28^{\prime} 11^{\prime \prime} \mathrm{N}, 007^{\circ} 25^{\prime} 04^{\prime \prime} \mathrm{W}$; alt. 400 m; 26.XI.2017; Andrade J. leg.; S (on Q. suber trunks and branches); Qs/Qr; Med.; UEVH-FUNGI 2004035. 


\section{Hebeloma collariatum Bruchet}

Figure 6E

Material examined. Newly recorded (n). PORTUGAL - Alentejo - SSMNP; 39²2'38"N, 007 23'13"W; alt. 520 m; 12.XII.2017; Andrade J. leg.; M; Ppn; Atl.; UEVHFUNGI 2003904.

Identification. This species can be recognized by its hazelnut-brown, convex to applanate pileus, darker in the centre and paler in the margin. Cream-coloured lamellae turn browner with maturity. Slender, fibrillose, whitish stipe, finely fibrillose at the top; veil often present. Raphanoid smell and bitter taste. Ellipsoid to subcitriform spores, slightly rugose. Cilindrical cheilocystidia.

Hebeloma crustuliniforme (Bull.) Quél.

Material examined. PORTUGAL - Alentejo • SSMNP; $39^{\circ} 27^{\prime} 12^{\prime \prime} \mathrm{N}, 007^{\circ} 26^{\prime} 33^{\prime \prime} \mathrm{W}$; alt. 436 m; 12.XI.2016; Andrade J. leg.; M; Qp/Qs; Med.; UEVH-FUNGI 2003598.

Hebeloma radicosum (Bull.) Ricken

Material examined. Newly recorded (n). PORTUGAL - Alentejo - SSMNP; 39 $18^{\prime} 03^{\prime \prime} \mathrm{N}, 007^{\circ} 24^{\prime} 43^{\prime \prime} \mathrm{W}$; alt. 680 m; 16.XI.2018; Andrade J. leg.; M; Qp/Qs; M/A; UEVHFUNGI 2004327.

\section{Hebeloma sinapizans (Paulet) Gillet}

Material examined. PORTUGAL - Alentejo • SSMNP; $39^{\circ} 23^{\prime} 56^{\prime \prime} \mathrm{N}, 007^{\circ} 25^{\prime} 34^{\prime \prime} \mathrm{W}$; alt. 580 m; 09.XII.2017; Andrade J. leg.; M; Qp/Qs; M/A; UEVH-FUNGI 2003900.

Hypholoma fasciculare (Huds.) P. Kumm.

Material examined. PORTUGAL - Alentejo • SSMNP; $39^{\circ} 20^{\prime} 03^{\prime \prime} \mathrm{N}, 007^{\circ} 24^{\prime} 33^{\prime \prime} \mathrm{W}$; alt. 690 m; 12.XII.2017; Andrade J. leg.; S; Qp/Cs; M/A; UEVH-FUNGI 2003890.

Inocybaceae

Inocybe acuta Boud.

Figure 6F

Material examined. Newly recorded (n). PORTUGAL - Alentejo • SSMNP; 39¹7'10"N, 007²0'57"W; alt. 770 m; 11.XI.2018; Andrade J. leg.; M; Ppi; Atl.; UEVHFUNGI 2004555.

Identification. This species can be recognized by its conical, conico-convex, or applanate pileus, with prominent acute or rather acute umbo. Dark brown or reddish brown, radially fibrillose pileipellis. Pale, grey to greybrown, ventricose, and moderately crowded lamellae. Slender stipe, slightly wider towards the base, sometimes ending in a small bulb, red-brown, dark brown, or yellow-brown, whitish at the base; usually faintly fibrillose or somewhat felted, pruinose at the top. Odour faint or slightly spermatic. Spores weakly nodulose with a few obtuse nodules or angles, rather dark yellow-brown. Inocybe acutella Bon has different spore morphology and habitat, and I. acutoides Kokkonen \& Vauras possesses a paler stipe and more obtuse umbo.
Inocybe fraudans (Britzelm.) Sacc.

Material examined. PORTUGAL - Alentejo - SSMNP; $39^{\circ} 27^{\prime} 12^{\prime \prime} \mathrm{N}, 007^{\circ} 26^{\prime} 33^{\prime \prime} \mathrm{W}$; alt. $440 \mathrm{~m}$; 12.XI.2016; Andrade J. leg.; M; Qp/Qs; Med.; UEVH-FUNGI 2003585.

\section{Inocybe geophylla (Bull.) P. Kumm.}

Material examined. PORTUGAL - Alentejo • SSMNP; $39^{\circ} 18^{\prime} 03^{\prime \prime} \mathrm{N}, 007^{\circ} 24^{\prime} 43^{\prime \prime} \mathrm{W}$; alt. $680 \mathrm{~m}$; 06.I.2016; Andrade J. leg.; M; Qp/Qs; M/A; UEVH-FUNGI 2003276.

\section{Phaeomarasmius erinaceus (Fr.) Scherff. ex Romagn.}

Material examined. PORTUGAL - Alentejo • SSMNP; $39^{\circ} 18^{\prime} 03^{\prime \prime} \mathrm{N}, 007^{\circ} 24^{\prime} 43^{\prime \prime} \mathrm{W}$; alt. $680 \mathrm{~m}$; 12.XI.2018; Andrade J. leg.; S; Qp/Qs; M/A; UEVH-FUNGI 2004484.

\section{Pseudosperma rimosum (Bull.) Matheny \& Esteve-Rav.}

Material examined. PORTUGAL - Alentejo • SSMNP; $39^{\circ} 27^{\prime} 18^{\prime \prime} \mathrm{N}, 007^{\circ} 26^{\prime} 35^{\prime \prime} \mathrm{W}$; alt. $430 \mathrm{~m}$; 12.XI.2016; Andrade J. leg.; M; Qs/Qr; Med.; UEVH-FUNGI 2003573.

Lyophyllaceae

\section{Lyophyllum transforme (Sacc.) Singer}

Material examined. Newly recorded (n). PORTUGAL - Alentejo • SSMNP; 39²0'03"N, 007²4'33"W; alt. 690 m; 01.XII.2019; Andrade J. leg.; S; Qp/Cs; M/A; UEVHFUNGI 2004799.

Meripilaceae

Meripilus giganteus (Pers.) P. Karst.

Figure 6G

Material examined. Newly recorded (n). PORTUGAL

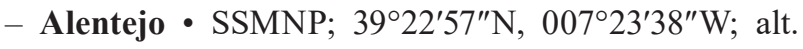
530 m; 22.X.2016; Andrade J. leg.; P; Fa; Atl.; UEVHFUNGI 2003537.

Identification. This polyporoid species can be recognized by the numerous rosette-like, flattened, fan-shaped pilei. Each pileus arises from a common stipe and can reach up to $30 \mathrm{~cm}$; upper pileus surface pale-brown to chestnut brown, fibrillose, darkening with maturation, and becoming zonate. Hymenium composed of clustered whitish tubes, which bruise brown or black. Spores ovoid and smooth. It may be confused with Laetiporus sulphureus (Bull.) Murrill, which displays yellow and orange colours and has a hymenium which does not bruise brown or black.

\section{Meruliaceae}

\section{Sarcodontia pachyodon (Pers.) Spirin}

Material examined. Newly recorded (n). PORTUGAL - Alentejo - SSMNP; 39²3'56"N, 007²5'34"W; alt. 580 m; 02.XII.2016; Andrade J. leg.; S; Qp/Qs; M/A; UEVHFUNGI 2003671. 
Mycenaceae

Mycena epipterygia (Scop.) Gray

Material examined. Newly recorded (n). PORTUGAL - Alentejo - SSMNP; $39^{\circ} 20^{\prime} 03^{\prime \prime} \mathrm{N}, 007^{\circ} 24^{\prime} 33^{\prime \prime} \mathrm{W}$; alt. 690 m; 27.XI.2016; Andrade J. leg.; S; Qp/Cs; M/A; UEVHFUNGI 2003643.

Mycena haematopus (Pers.) P. Kumm.

Material examined. PORTUGAL - Alentejo - SSMNP; $39^{\circ} 23^{\prime} 44^{\prime \prime} \mathrm{N}, 007^{\circ} 25^{\prime} 09^{\prime \prime} \mathrm{W}$; alt. 580 m; 06.XI.2014; Andrade J. leg.; S; Qp/Qs; M/A; UEVH-FUNGI 2002978.

Mycena inclinata (Fr.) Quél.

Material examined. Newly recorded (n). PORTUGAL - Alentejo - SSMNP; 39 23'19"N, 007 $25^{\prime} 21^{\prime \prime} \mathrm{W}$; alt. 620 m; 12.XII.2017; Andrade J. leg.; S; Qp/Cs; M/A; UEVHFUNGI 2003892

Mycena polygramma (Bull.) Gray

Material examined. Newly recorded (n). PORTUGAL - Alentejo - SSMNP; 39²2'28"N, 007²2'08"W; alt. 720 m; 09.XII.2017; Andrade J. leg.; S; Ppi; M/A; UEVH-FUNGI 2003909

\section{Mycena pura (Pers.) P. Kumm.}

Material examined. PORTUGAL - Alentejo • SSMNP; $39^{\circ} 19^{\prime} 15^{\prime \prime} \mathrm{N}, 007^{\circ} 24^{\prime} 47^{\prime \prime} \mathrm{W}$; alt. 600 m; 12.XI.2016; Andrade J. leg.; S; Qp/Qs; M/A; UEVH-FUNGI 2003605.

\section{Mycena rosea Gramberg}

Material examined. PORTUGAL - Alentejo - SSMNP $39^{\circ} 23^{\prime} 19^{\prime \prime} \mathrm{N}, 007^{\circ} 25^{\prime} 21^{\prime \prime} \mathrm{W}$; alt. 620 m; 12.XI.2016; Andrade J. leg.; S; Qp/Cs; M/A; UEVH-FUNGI 2003607.

Mycena seynii Quél.

Material examined. PORTUGAL - Alentejo - SSMNP; $39^{\circ} 17^{\prime} 15^{\prime \prime} \mathrm{N}, 007^{\circ} 21^{\prime} 18^{\prime \prime} \mathrm{W}$; alt. 770 m; 19.XI.2013; Andrade J. leg.; S (on fallen Pinus pinaster cones); Ppi; M/A; UEVH-FUNGI 2002556.

Omphalotaceae

\section{Collybiopsis quercophila (Pouzar) R.H. Petersen}

Material examined. PORTUGAL - Alentejo • SSMNP; $39^{\circ} 19^{\prime} 15^{\prime \prime} \mathrm{N}, 007^{\circ} 24^{\prime} 47^{\prime \prime} \mathrm{W}$; alt. 600 m; 03.XI.2015; Andrade J. leg.; S (on Quercus spp. leaf litter); Qp/Qs; M/A; UEVH-FUNGI 2003424.

\section{Gymnopus dryophilus (Bull.) Murrill}

Material examined. PORTUGAL - Alentejo • SSMNP; $39^{\circ} 17^{\prime} 15^{\prime \prime} \mathrm{N}, 007^{\circ} 21^{\prime} 18^{\prime \prime} \mathrm{W}$; alt. $770 \mathrm{~m}$; 06.XI.2018; Andrade J. leg.; S; Ppi; M/A; UEVH-FUNGI 2004385.

\section{Omphalotus illudens (Schwein.) Bresinsky \& Besl} Figure 6H

Material examined. Newly recorded (n). PORTUGAL -Alentejo • SSMNP; 39²4'23"N, 007 24'31"W; alt. 660 m; 11.XI.2018; Andrade J. leg.; S (on C. sativa trunks); Qp/Cs; M/A; UEVH-FUNGI 2004287.

Identification. This species can be recognized by its, convex to funnel-shaped pileus, dry, radially fibrillose, and yellowish to orange-brown pileipellis. Lamellae decurrent, thin, and arched. Stipe long, fibrous, sinuous, and fasciculate; yellow or orange but paler than the pileus. This species can be distinguished from $O$. olearius (DC.) Singer by its lighter yellowish hues and different habitat.

\section{Omphalotus olearius (DC.) Singer}

Material examined. PORTUGAL - Alentejo • SSMNP; $39^{\circ} 08^{\prime} 15^{\prime \prime} \mathrm{N}, 007^{\circ} 12^{\prime} 54^{\prime \prime} \mathrm{W}$; alt. 370 m; 20.X.2014; Andrade J. leg.; S (on buried roots of Quercus spp.); Qs/Qr; M/A; UEVH-FUNGI 2002874.

\section{Rhodocollybia butyracea (Bull.) Lennox}

Material examined. PORTUGAL - Alentejo - SSMNP; $39^{\circ} 19^{\prime} 15^{\prime \prime} \mathrm{N}, 007^{\circ} 24^{\prime} 47^{\prime \prime} \mathrm{W}$; alt. 600 m; 08.XII.2017; Andrade J. leg.; S; Qp/Qs; M/A; UEVH-FUNGI 2003912.

Paxillaceae

\section{Paxillus involutus (Batsch) Fr.}

Material examined. PORTUGAL - Alentejo • SSMNP; $39^{\circ} 18^{\prime} 05^{\prime \prime} \mathrm{N}, 007^{\circ} 24^{\prime} 40^{\prime \prime} \mathrm{W}$; alt. $700 \mathrm{~m}$; 30.XI.2015; Andrade J. leg.; M; Qp/Cs; M/A; UEVH-FUNGI 2003162.

Phallaceae

Clathrus ruber P. Micheli ex Pers.

Material examined. PORTUGAL - Alentejo - SSMNP; $39^{\circ} 29^{\prime} 45^{\prime \prime} \mathrm{N}, 007^{\circ} 27^{\prime} 03^{\prime \prime} \mathrm{W}$; alt. $370 \mathrm{~m}$; 19.II.2020; Andrade J. leg.; S; Qs/Qr; Med.; UEVH-FUNGI 2004903.

\section{Phallus impudicus L.}

Material examined. PORTUGAL - Alentejo • SSMNP; $39^{\circ} 23^{\prime} 09^{\prime \prime} \mathrm{N}, 007^{\circ} 21^{\prime} 42^{\prime \prime} \mathrm{W}$; alt. 640 m; 01.XII.2019; Andrade J. leg.; S; Qp/Qs; M/A; UEVH-FUNGI 2004825.

Physalacriaceae

Desarmillaria tabescens (Scop.) R.A. Koch \& Aime Material examined. PORTUGAL - Alentejo - SSMNP; $39^{\circ} 23^{\prime} 56^{\prime \prime} \mathrm{N}, 007^{\circ} 25^{\prime} 34^{\prime \prime} \mathrm{W}$; alt. 580 m; 15.XII.2015; Andrade J. leg.; P; Qp/Qs; M/A; UEVH-FUNGI 2003194.

\section{Xerula pudens (Pers.) Singer}

Material examined. Newly recorded (n). PORTUGAL - Alentejo • SSMNP; 39 $19^{\prime} 15^{\prime \prime} \mathrm{N}, 007^{\circ} 24^{\prime} 47^{\prime \prime} \mathrm{W}$; alt. 600 m; 11.XI.2018; Andrade J. leg.; S; Qp/Qs; Med.; UEVHFUNGI 2003912.

\section{Pleurotaceae}

Pleurotus ostreatus (Jacq.) P. Kumm.

Material examined. PORTUGAL - Alentejo - SSMNP; $39^{\circ} 18^{\prime} 03^{\prime \prime} \mathrm{N}, 007^{\circ} 24^{\prime} 43^{\prime \prime} \mathrm{W}$; alt. $680 \mathrm{~m}$; 15.XI.2016; 
Andrade J. leg.; S; Qp/Qs; M/A; UEVH-FUNGI 2003617.

Pluteaceae

\section{Pluteus pouzarianus Singer}

Material examined. Newly recorded (n). PORTUGAL - Alentejo • SSMNP; 39॰17'10"N, 007²0'57"W; alt. 770 m; 09.XII.2017; Andrade J. leg.; S (on P. pinaster decaying wood); Ppi; Atl.; UEVH-FUNGI 2003898.

Pluteus salicinus (Pers.) P. Kumm.

Material examined. PORTUGAL - Alentejo • SSMNP; $39^{\circ} 22^{\prime} 57^{\prime \prime} \mathrm{N}, 007^{\circ} 22^{\prime} 55^{\prime \prime} \mathrm{W}$; alt. $510 \mathrm{~m}$; 28.X.2016; Andrade J. leg.; S; Ppn; Atl.; UEVH-FUNGI 2003580.

Volvopluteus gloiocephalus (DC.) Vizzini, Contu \& Justo

Material examined. PORTUGAL - Alentejo - SSMNP; $39^{\circ} 22^{\prime} 48^{\prime \prime} \mathrm{N}, 007^{\circ} 23^{\prime} 18^{\prime \prime} \mathrm{W}$; alt. 520 m; 12.XI.2018; Andrade J. leg.; S; Ppa; M/A; UEVH-FUNGI 2004479.

Polyporaceae

Ganoderma resinaceum Boud.

Material examined. PORTUGAL - Alentejo • SSMNP; $39^{\circ} 22^{\prime} 56^{\prime \prime} \mathrm{N}, 007^{\circ} 22^{\prime} 40^{\prime \prime} \mathrm{W}$; alt. 520 m; 01.XII.2015; Andrade J. leg.; P; Ppn; Atl.; UEVH-FUNGI 2003165.

Lentinus arcularius (Batsch) Zmitr.

Material examined. PORTUGAL - Alentejo • SSMNP; $39^{\circ} 24^{\prime} 23^{\prime \prime} \mathrm{N}, 007^{\circ} 24^{\prime} 31^{\prime \prime} \mathrm{W}$; alt. $660 \mathrm{~m}$; 27.IV.2016; Andrade J. leg.; S; Qp/Cs; M/A; UEVH-FUNGI 2003410.

\section{Neofavolus alveolares (DC.) Sotome \& T. Hatt.}

Material examined. PORTUGAL - Alentejo • SSMNP; $39^{\circ} 10^{\prime} 11^{\prime \prime} \mathrm{N}, 007^{\circ} 09^{\prime} 37^{\prime \prime} \mathrm{W}$; alt. 350 m; 02.XII.2017; Andrade J. leg.; S; Qs/Qr; M/A; UEVH-FUNGI 2004037.

Panus neostrigosus Drechsler-Santos \& Wartchow Material examined. PORTUGAL - Alentejo • SSMNP; $39^{\circ} 23^{\prime} 56^{\prime \prime} \mathrm{N}, 007^{\circ} 25^{\prime} 34^{\prime \prime} \mathrm{W}$; alt. 580 m; 13.XI.2013; Andrade J. leg.; S; Qp/Qs; M/A; UEVH-FUNGI 2002546.

\section{Pycnoporus cinnabarinus (Jacq.) P. Karst.}

Figure 6I

Material examined. Newly recorded (n). PORTUGAL - Alentejo - SSMNP; 39 23'56"N, 007²5'34"W; alt. 580 m; 09.XI.2015; Andrade J. leg.; S; Qp/Qs; M/A; UEVHFUNGI 2003108.

Identification. This species can be recognized by its bright orange, semicircular to kidney-shaped basidiocarps, pubescent and rough pileipellis. Hymenium with short tubes concolourous with the pilepellis and rounded-angular pores, bright cinnabar red, often elongated or labyrinthine. Pycnoporus sanguineus (L.) Murrill is thinner and more brightly coloured and features a shinier surface.
Trametes versicolor (L.) Lloyd

Material examined. PORTUGAL - Alentejo - SSMNP; $39^{\circ} 23^{\prime} 09^{\prime \prime} \mathrm{N}, 007^{\circ} 21^{\prime} 42^{\prime \prime} \mathrm{W}$; alt. $640 \mathrm{~m}$; 21.X.2016; Andrade J. leg.; S; Qp/Qs; M/A; UEVH-FUNGI 2003729.

Psathyrellaceae

\section{Coprinopsis picacea (Bull.) Redhead, Vilgalys \&} Moncalvo

Material examined. PORTUGAL - Alentejo - SSMNP; $39^{\circ} 19^{\prime} 15^{\prime \prime} \mathrm{N}, 007^{\circ} 24^{\prime} 47^{\prime \prime} \mathrm{W}$; alt. $600 \mathrm{~m}$; 13.X.2019; Andrade J. leg.; S; Qp/Qs; M/A; UEVH-FUNGI 2004755.

\section{Psathyrella candolleana (Fr.) Maire}

Material examined. PORTUGAL - Alentejo - SSMNP; $39^{\circ} 27^{\prime} 12^{\prime \prime} \mathrm{N}, 007^{\circ} 26^{\prime} 33^{\prime \prime} \mathrm{W}$; alt. $440 \mathrm{~m}$; 03.XI.2015; Andrade J. leg.; S; Qp/Qs; M/A; UEVH-FUNGI 2003074.

\section{Psathyrella murcida (Fr.) Kits van Wav.}

Material examined. PORTUGAL - Alentejo • SSMNP; $39^{\circ} 18^{\prime} 03^{\prime \prime} \mathrm{N}, 007^{\circ} 24^{\prime} 43^{\prime \prime} \mathrm{W}$; alt. $680 \mathrm{~m}$; 11.XI.2018; Andrade J. leg.; S; Qp/Qs; Atl.; UEVH-FUNGI 2004395.

\section{Rickenella fibula (Bull.) Raithelh.}

Material examined. PORTUGAL - Alentejo • SSMNP; $39^{\circ} 23^{\prime} 09^{\prime \prime} \mathrm{N}, 007^{\circ} 21^{\prime} 42^{\prime \prime} \mathrm{W}$; alt. 640 m; 17.XI.2015; Andrade J. leg.; S; Qp/Qs; M/A; UEVH-FUNGI 2003129.

Russulaceae

\section{Lactarius camphoratus (Bull.) Fr.}

Material examined. PORTUGAL - Alentejo • SSMNP; $39^{\circ} 23^{\prime} 56^{\prime \prime} \mathrm{N}, 007^{\circ} 25^{\prime} 34^{\prime \prime} \mathrm{W}$; alt. $580 \mathrm{~m}$; 17.XI.2015; Andrade J. leg.; M; Qp/Qs; M/A; UEVH-FUNGI 2003130.

\section{Lactarius chrysorrheus Fr.}

Material examined. PORTUGAL - Alentejo - SSMNP; 39 $23^{\prime} 44^{\prime \prime} \mathrm{N}, 007^{\circ} 25^{\prime} 09^{\prime \prime} \mathrm{W}$; alt. 580 m; 09.XI.2015; Andrade J. leg.; M; Qp/Qs; M/A; UEVH-FUNGI 2003107.

\section{Lactarius cistophilus Bon \& Trimbach}

Material examined. PORTUGAL - Alentejo - SSMNP; $39^{\circ} 30^{\prime} 46^{\prime \prime} \mathrm{N}, 007^{\circ} 28^{\prime} 43^{\prime \prime} \mathrm{W}$; alt. 310 m; 17.XI.2015; Andrade J. leg.; M; S; Med.; UEVH-FUNGI 2003147.

\section{Lactarius controversus Pers.}

Material examined. Newly recorded (n). PORTUGAL

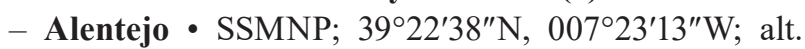
520 m; 15.X.2016; Andrade J. leg.; M; Ppn; Atl.; UEVHFUNGI 2003535.

\section{Lactarius deliciosus (L.) Gray}

Material examined. PORTUGAL - Alentejo - SSMNP; $39^{\circ} 22^{\prime} 28^{\prime \prime} \mathrm{N}, 007^{\circ} 22^{\prime} 08^{\prime \prime} \mathrm{W}$; alt. 720 m; 09.XI.2015; Andrade J. leg.; M; Ppi; M/A; UEVH-FUNGI 2003081.

Lactarius hepaticus Plowr.

Material examined. PORTUGAL - Alentejo • SSMNP; 
$39^{\circ} 18^{\prime} 06^{\prime \prime} \mathrm{N}, 007^{\circ} 24^{\prime} 10^{\prime \prime} \mathrm{W}$; alt. 700 m; 01.XII.2017; Andrade J. leg.; M; Ppi; Med.; UEVH-FUNGI 2004116.

Lactarius scrobiculatus (Scop.) Fr.

Material examined. Newly recorded (n). PORTUGAL - Alentejo - SSMNP; 39²2'28"N, 007²2'08"W; alt. 720 m; 025XI.2016; Andrade J. leg.; M; Ppi; M/A; UEVH-FUNGI 2003545.

\section{Lactarius tesquorum Malençon}

Material examined. PORTUGAL - Alentejo • SSMNP; $39^{\circ} 30^{\prime} 46^{\prime \prime} \mathrm{N}, 007^{\circ} 28^{\prime} 43^{\prime \prime} \mathrm{W}$; alt. 310 m; 01.XII.2015; Andrade J. leg.; M; S; Med.; UEVH-FUNGI 2003166.

Lactarius zonarius (Bull.) Fr.

Material examined. PORTUGAL - Alentejo • SSMNP; $39^{\circ} 18^{\prime} 15^{\prime \prime} \mathrm{N}, 007^{\circ} 24^{\prime} 35^{\prime \prime} \mathrm{W}$; alt. 720 m; 04.XI.2019; Andrade J. leg.; M; Qp/Cs; Atl.; UEVH-FUNGI 2004768.

\section{Lactifluus piperatus (L.) Roussel}

Material examined. PORTUGAL - Alentejo • SSMNP; $39^{\circ} 20^{\prime} 03^{\prime \prime} \mathrm{N}, 007^{\circ} 24^{\prime} 33^{\prime \prime} \mathrm{W}$; alt. $690 \mathrm{~m}$; 05.XII.2013; Andrade J. leg.; M; Qp/Cs; M/A; UEVH-FUNGI 2002666.

\section{Lactifluus rugatus (Kühner \& Romagn.) Verbeken}

Material examined. PORTUGAL - Alentejo • SSMNP; $39^{\circ} 27^{\prime} 18^{\prime \prime} \mathrm{N}, 007^{\circ} 26^{\prime} 35^{\prime \prime} \mathrm{W}$; alt. 430 m; 24.XI.2015; Andrade J. leg.; M; Qs/Qr; Med.; UEVH-FUNGI 2003030.

\section{Lactifluus volemus (Fr.) Kuntze}

Material examined. PORTUGAL - Alentejo • SSMNP; $39^{\circ} 23^{\prime} 56^{\prime \prime} \mathrm{N}, 007^{\circ} 25^{\prime} 34^{\prime \prime} \mathrm{W}$; alt. $580 \mathrm{~m}$; 02.I.2018; Andrade J. leg.; M; Qp/Qs; M/A; UEVH-FUNGI 2004114.

\section{Russula acrifolia Romagn.}

Material examined. Newly recorded (n). PORTUGAL - Alentejo • SSMNP; 39¹9'15"N, 007²4'47"W; alt. 600 m; 12.XI.2016; Andrade J. leg.; M; Qp/Qs; M/A; UEVHFUNGI 2003587.

\section{Russula amethystina Quél.}

Material examined. Newly recorded (n). PORTUGAL - Alentejo - SSMNP; 39 $19^{\prime} 17^{\prime \prime} \mathrm{N}, 007^{\circ} 23^{\prime} 08^{\prime \prime} \mathrm{W}$; alt. 770 m; 19.XI.2018; Andrade J. leg.; M; Ppi; Atl.; UEVHFUNGI 2004322.

\section{Russula amoenicolor Romagn.}

Material examined. PORTUGAL - Alentejo - SSMNP; $39^{\circ} 23^{\prime} 56^{\prime \prime} \mathrm{N}, 007^{\circ} 25^{\prime} 34^{\prime \prime} \mathrm{W}$; alt. 580 m; 05.XI.2016; Andrade J. leg.; M; Qp/Qs; M/A; UEVH-FUNGI 2003547.

Russula atropurpurea (Krombh.) Britzelm.

Material examined. Newly recorded (n). PORTUGAL - Alentejo - SSMNP; 39 $23^{\prime} 56^{\prime \prime} \mathrm{N}, 007^{\circ} 25^{\prime} 34^{\prime \prime} \mathrm{W}$; alt. 580 m; 06.XII.2019; Andrade J. leg.; M; Qp/Qs; M/A; UEVH-FUNGI 2004793.

\section{Russula aurea Pers}

Material examined. PORTUGAL - Alentejo - SSMNP; $39^{\circ} 23^{\prime} 19^{\prime \prime} \mathrm{N}, 007^{\circ} 25^{\prime} 21^{\prime \prime} \mathrm{W}$; alt. $620 \mathrm{~m}$; 24.XI.2016; Andrade J. leg.; M; Qp/Cs; M/A; UEVH-FUNGI 2003616.

\section{Russula chloroides (Krombh.) Bres.}

Material examined. PORTUGAL - Alentejo • SSMNP; $39^{\circ} 27^{\prime} 18^{\prime \prime} \mathrm{N}, 007^{\circ} 26^{\prime} 35^{\prime \prime} \mathrm{W}$; alt. $430 \mathrm{~m}$; 20.X.2019; Andrade J. leg.; M; Qs/Qr; M/A; UEVH-FUNGI 2004750

Russula cyanoxantha (Schaeff.) Fr.

Material examined. PORTUGAL - Alentejo - SSMNP; $39^{\circ} 23^{\prime} 44^{\prime \prime} \mathrm{N}, 007^{\circ} 25^{\prime} 09^{\prime \prime} \mathrm{W}$; alt. 580 m; 20.X.2019; Andrade J. leg.; M; Qp/Qs; M/A; UEVH-FUNGI 2004758.

\section{Russula delica Fr.}

Material examined. PORTUGAL - Alentejo • SSMNP; $39^{\circ} 19^{\prime} 15^{\prime \prime} \mathrm{N}, 007^{\circ} 24^{\prime} 47^{\prime \prime} \mathrm{W}$; alt. 600 m; 05.XI.2016; Andrade J. leg.; M; Qp/Qs; M/A; UEVH-FUNGI 2003559.

\section{Russula farinipes Romell}

Figure 6J

Material examined. Newly recorded (n). PORTUGAL - Alentejo • SSMNP; $39^{\circ} 18^{\prime} 15^{\prime \prime} \mathrm{N}, 007^{\circ} 24^{\prime} 35^{\prime \prime} \mathrm{W}$; alt. 720 m; 02.I.2019; Andrade J. leg.; M; Qp/Cs; Atl.; UEVHFUNGI 2004708.

Identification. This species can be recognized by the initially convex, then applanate, and finally depressed pileus, yellow to light ochraceous; slightly viscid pileipellis. Dense but well-spaced lamellae. Stipe cylindrical, initially firm and full, then finally hollow, white, with some yellow spots towards the base; with a pruinose surface on the apex due to the presence of caulocystidia. Slightly fruity smell and distinctly spicy flavour. Pure white sporata and small elliptical spores, with small, pointed warts without connections. Russula fellea is recognized by its larger size and totally different microscopy; $R$. solaris is distinguished by its more markedly yellow cap and its fragile consistency.

\section{Russula grata Britzelm.}

Material examined. Newly recorded (n). PORTUGAL

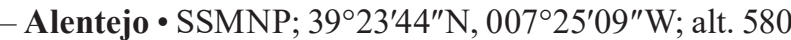
m; 10.IV.2018; Andrade J. leg.; M; Qp/Qs; M/A; UEVHFUNGI 2004163.

\section{Russula heterophylla (Fr.) Fr.}

Material examined. PORTUGAL - Alentejo - SSMNP; $39^{\circ} 19^{\prime} 15^{\prime \prime} \mathrm{N}, 007^{\circ} 24^{\prime} 47^{\prime \prime} \mathrm{W}$; alt. 600 m; 12.IV.2018; Andrade J. leg.; M; Qp/Qs; M/A; UEVH-FUNGI 2004164.

\section{Russula illota Romagn.}

Material examined. Newly recorded (n). PORTUGAL - Alentejo - SSMNP; 39²3'56"N, 007²5'34"W; alt. 580 m; 05.XI.2016; Andrade J. leg.; M; Qp/Qs; M/A; UEVHFUNGI 2003570. 


\section{Russula melitodes Romagn.}

Material examined. Newly recorded (n). PORTUGAL

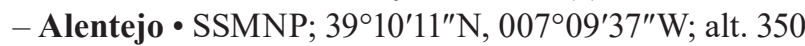
m; 27.X.2016; Andrade J. leg.; M; Qs/Qr; Med.; UEVHFUNGI 2003562.

\section{Russula olivacea (Schaeff.) Fr.}

Material examined. PORTUGAL - Alentejo • SSMNP; $39^{\circ} 10^{\prime} 11^{\prime \prime} \mathrm{N}, 007^{\circ} 09^{\prime} 37^{\prime \prime} \mathrm{W}$; alt. 350 m; 26.XI.2019; Andrade J. leg.; M; Qs/Qr; Med.; UEVH-FUNGI 2004801.

\section{Russula oreades Sarnari}

Figure 6K

Material examined. Newly recorded (n). PORTUGAL - Alentejo - SSMNP 39²9'45"N, 007²7'03"W; alt. 370 m; 20.X.2019; Andrade J. leg.; M; Qs/Qr; Med.; UEVHFUNGI 2004765.

Identification. This species can be recognized by the applanate pileus, often with a central depression, lobed and striate wavy margins, and velvety purple red pileipellis, with varying brown, pink and violet tinges. Thick, wellspaced, white lamellae turning butter yellow with spore maturation and with visible interconnected veins. Stipe cylindrical to irregularly clavate-curved, white, finely wrinkled, initially firm then spongy. Faint fruity smell that becomes honey-like in dry specimens; taste mild. Yellowish sporata and broadly ellipsoidal to subglobose spores with small, interconnected warts forming a thin, often incomplete network. Russula prinophila is very similar but has thicker lamellae and more yellowing flesh.

\section{Russula parodorata Sarnari}

Material examined. PORTUGAL - Alentejo - SSMNP $39^{\circ} 27^{\prime} 12^{\prime \prime} \mathrm{N}$, $007^{\circ} 26^{\prime} 33^{\prime \prime} \mathrm{W}$; alt. 440 m; 21.XI.2018; Andrade J. leg.; M; Qp/Qs; Med.; UEVH-FUNGI 2004554.

\section{Russula praetervisa Sarnari}

Material examined. PORTUGAL - Alentejo • SSMNP; $39^{\circ} 27^{\prime} 12^{\prime \prime} \mathrm{N}, 007^{\circ} 26^{\prime} 33^{\prime \prime} \mathrm{W}$; alt. $440 \mathrm{~m}$; 01.XI.2018; Andrade J. leg.; M; Qp/Qs; Med.; UEVH-FUNGI 2004371.

\section{Russula risigallina (Batsch) Sacc.}

Material examined. Newly recorded (n). PORTUGAL - Alentejo - SSMNP; 39¹9'17"N, 007²4'54"W; alt. 600 m; 10.V.2018; Andrade J. leg.; M; Qp/Cs; Med.; UEVHFUNGI 2004135.

\section{Russula rosea Pers.}

Material examined. PORTUGAL - Alentejo • SSMNP; $39^{\circ} 24^{\prime} 23^{\prime \prime} \mathrm{N}, 007^{\circ} 24^{\prime} 31^{\prime \prime} \mathrm{W}$; alt. $660 \mathrm{~m}$; 09.XI.2015; Andrade J. leg.; M; Qp/Cs; M/A; UEVH-FUNGI 2003263.

\section{Russula sericatula Romagn.}

Figure 6L

Material examined. Newly recorded (n). PORTUGAL - Alentejo • SSMNP; 39 $18^{\prime} 15^{\prime \prime} \mathrm{N}, 007^{\circ} 24^{\prime} 35^{\prime \prime} \mathrm{W}$; alt. 720 m; 12.III.2020; Andrade J. leg.; M; Qp/Cs; Atl.; UEVHFUNGI 2004902.
Identification. This robust species can be recognized by the initially subhemispherical, then applanate pileus, often with a central depression, greenish-yellow in the centre and purple-red on the margin, mat, not striate. Stipe robust, white, cylindrical, wrinkled. Orange sporata and obovate to ellipsoidal spores with isolated spiny warts. Primordial hyphae encrusted. Mild flavour, odourless. Similar to $R$. melitodes which has a slightly honey-like odour.

\section{Russula silvestris (Singer) Reumaux \\ Figure 7A}

Material examined. Newly recorded (n). PORTUGAL - Alentejo - SSMNP; 39 24'23"N, 007 24'31"W; alt. 660 m; 20.X.2019; Andrade J. leg.; M; Qp/Cs; M/A; UEVHFUNGI 2004767.

Identification. This species can be recognized by the subglobose, then finally expanded pileus, with slightly depressed centre and smooth margins; shiny to slightly opaque, cherry-red or red-pink pileipellis, more or less washed up to pinkish-whitish. Moderately dense lamellae, first white then whitish with spore maturation. Stipe cylindrical or with flared apex and slightly enlarged base, fragile, smooth or finely pruinose, immutable white. Very distinct coconut milk-like smell and spicy flavour. Whitish sporata and obovoid spores, with warts isolated or united in short ridges, forming a thin, incomplete network. Russula rhodomelanea is redder, less slender, and blackens when bruised.

\section{Russula sororia (Fr.) Romell}

Material examined. PORTUGAL - Alentejo • SSMNP; $39^{\circ} 23^{\prime} 56^{\prime \prime} \mathrm{N}, 007^{\circ} 25^{\prime} 34^{\prime \prime} \mathrm{W}$; alt. 580 m; 12.XI.2016; Andrade J. leg.; M; Qp/Qs; M/A; UEVH-FUNGI 2003601.

\section{Russula torulosa Bers.}

Material examined. PORTUGAL - Alentejo - SSMNP; $39^{\circ} 17^{\prime} 15^{\prime \prime} \mathrm{N}, 007^{\circ} 21^{\prime} 18^{\prime \prime} \mathrm{W}$; alt. $770 \mathrm{~m}$; 26.XI.2016; Andrade J. leg.; M; Ppi; M/A; UEVH-FUNGI 2003683.

\section{Russula turci Bers. \\ Figure 7B}

Material examined. Newly recorded (n). PORTUGAL - Alentejo - SSMNP; 39 $17^{\prime} 10^{\prime \prime} \mathrm{N}, 007^{\circ} 20^{\prime} 57^{\prime \prime} \mathrm{W}$; alt. 770 m; 03.XII.2016; Andrade J. leg.; M; Ppi; M/A; UEVHFUNGI 2003691.

Identification. This species can be recognized by its violet to lilac pileipellis and conspicuous odour. Pileus very viscid, initially convex then slightly umbonate and discoloured at the centre. Lamellae adnate, crowded, white to yellow, with brown spots at maturity. Stipe cylindrical, slightly enlarged at base, white to pinkish, often pruinose. Dark yellow sporata and ellipsoid spores, usually united in short ridges, never forming a complete reticulum. Mild flavour and strong odour of iodine. This species can be distinguished from other violet Russula species in having its pileus discoloured at the centre and its iodoform odour, particularly strong near the stipe base. 


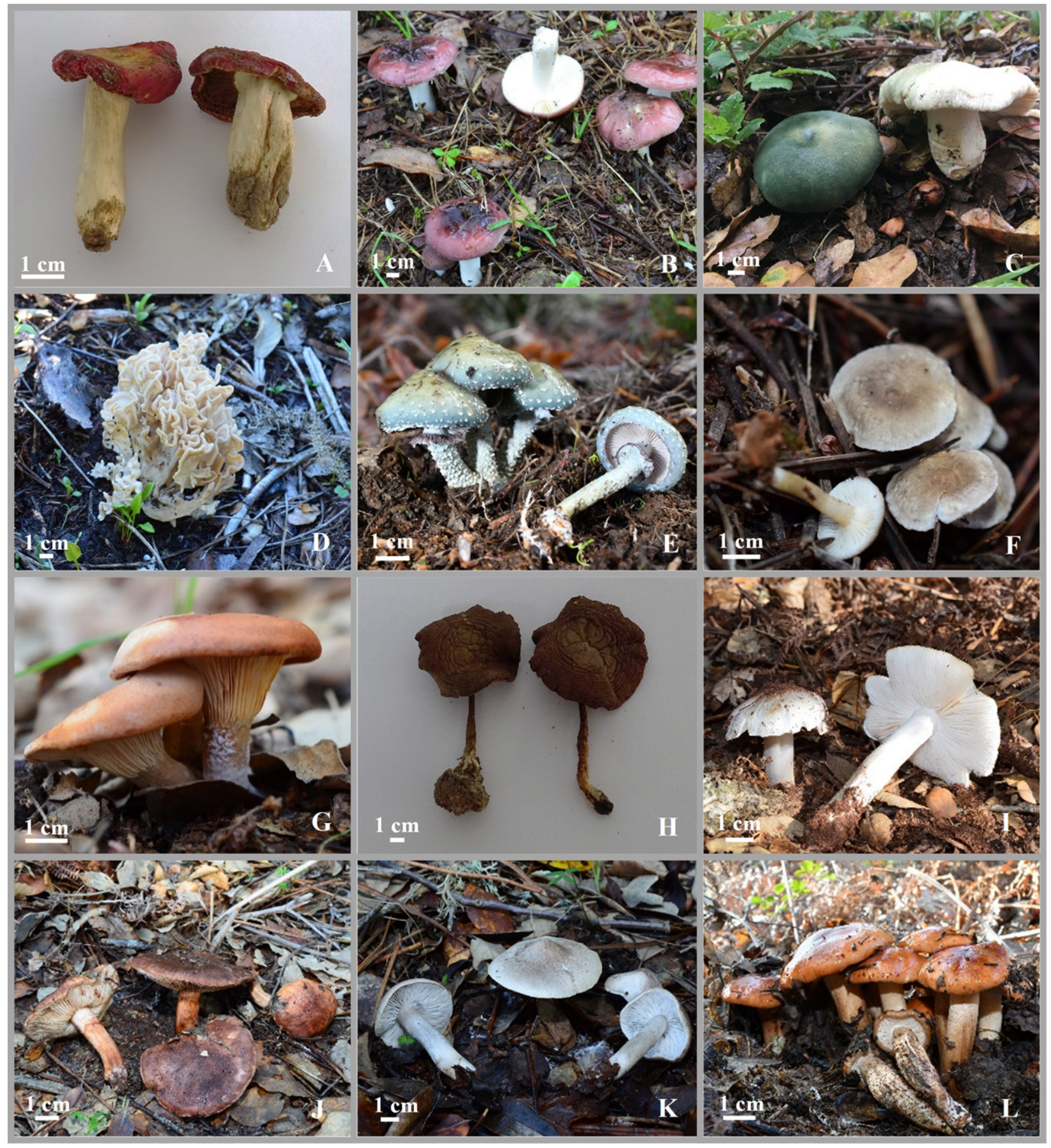

Figure 7. A. Russula silvestris (Singer) Reumaux** B. Russula turci Bers.* C. Russula virescens (Schaeff.) Fr.* D. Sparassis crispa (Wulfen) Fr.* E. Stropharia aeruginosa (Curtis) Quél.* F. Cantharellula umbonata (J. F. Gmel.) Singer** G. Clitocybe bresadolana Singer** H. Melanoleuca polioleuca (Fr.) Kühner \& Maire** I. Tricholoma columbetta (Fr.) P. Kumm.* J. Tricholoma imbricatum (Fr.) P. Kumm. ${ }^{*}$ K. Tricholoma scalpturatum (Fr.) Quél.* L. Tricholoma stans (Fr.) Sacc.. ${ }^{*}{ }^{*}=$ newly recorded from Alto Alentejo Province; ${ }^{* *}=$ newly recorded from continental Portugal)

Russula violeipes Quél.

Material examined. Newly recorded (n). PORTUGAL - Alentejo • SSMNP; $39^{\circ} 20^{\prime} 03^{\prime \prime} \mathrm{N}, 007^{\circ} 24^{\prime} 33^{\prime \prime} \mathrm{W}$; alt. 690 m; 12.XI.2016; Andrade J. leg.; M; Qp/Cs; M/A; UEVHFUNGI 2003619.

Russula virescens (Schaeff.) Fr.

Figure 7C

Material examined. Newly recorded (n). PORTUGAL

- Alentejo • SSMNP; 39 24'23"N, 007²4'31"W; alt. 660 m; 10.V.2018; Andrade J. leg.; M; Qp/Cs; M/A; UEVHFUNGI 2004161.

Identification. This species can be recognized by its usually cracked, greenish pileipellis, paler at the pileus margin. Pileus initially smooth, soon cracking into several small patches, convex to applanate, slightly umbonate at the centre, up to $10 \mathrm{~cm}$ in diameter. Lamellae crowded, adnate, cream coloured. Stipe cylindrical to fusiform, tapering at the base, white to cream, smooth. Taste mild and nutty. Sporata cream, spores ellipsoidal 
to ovoid, with small warts, occasionally interconnected. This species can be discerned from $R$. aeruginea Lindblad ex Fr. which does not have a cracked pileus.

Sclerodermataceae

Scleroderma polyrhizum (J.F. Gmel.) Pers.

Material examined. PORTUGAL - Alentejo • SSMNP; $39^{\circ} 19^{\prime} 17^{\prime \prime} \mathrm{N}, 007^{\circ} 23^{\prime} 08^{\prime \prime} \mathrm{W}$; alt. 770 m; 19.XI.2018; Andrade J. leg.; M; Ppi; Atl.; UEVH-FUNGI 2004317.

Scleroderma verrucosum (Bull.) Pers.

Material examined. PORTUGAL - Alentejo - SSMNP; $39^{\circ} 08^{\prime} 15^{\prime \prime} \mathrm{N}, 007^{\circ} 12^{\prime} 54^{\prime \prime} \mathrm{W}$; alt. 370 m; 30.XI.2015; Andrade J. leg.; M; Qs/Qr; Med.; UEVH-FUNGI 2003164.

Sparassidaceae

Sparassis crispa (Wulfen) Fr. Figure 7D

Material examined. Newly recorded (n). PORTUGAL

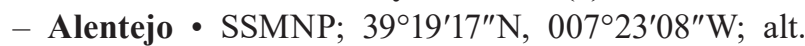
770 m; 30.X.2016; Andrade J. leg.; P; Ppi; Atl.; UEVHFUNGI 2003549.

Identification. This species can be recognized by the irregular globose basidiocarps, up to $25 \mathrm{~cm}$ tall and $40 \mathrm{~cm}$ in diameter, composed by several flat and wavy lobes, creamy white when young, becoming brown with age. Faint sweet smell, taste of hazelnuts. Spores broadly ellipsoidal to ovoid. This species can be discerned from S. spathulata (Schwein.) Fr. which has erect, straight lobes, and from Grifola frondosa (Dicks.) Gray which has pores on the underside of the lobes, a different colour range, and different ecology.

Stereaceae

Stereum hirsutum (Willd.) Pers.

Material examined. PORTUGAL - Alentejo • SSMNP; $39^{\circ} 24^{\prime} 23^{\prime \prime} \mathrm{N}, 007^{\circ} 24^{\prime} 31^{\prime \prime} \mathrm{W}$; alt. $660 \mathrm{~m}$; 22.X.2013; Andrade J. leg.; S; Qp/Cs; M/A; UEVH-FUNGI 2002254.

Strophariaceae

Cyclocybe aegerita (V. Brig.) Vizzini

Material examined. PORTUGAL - Alentejo • SSMNP; $39^{\circ} 22^{\prime} 56^{\prime \prime} \mathrm{N}, 007^{\circ} 22^{\prime} 40^{\prime \prime} \mathrm{W}$; alt. $520 \mathrm{~m}$; 24.II.2020; Andrade J. leg.; S; Ppn; M/A; UEVH-FUNGI 2004892.

\section{Stropharia aeruginosa (Curtis) Quél.}

Figure 7E

Material examined. Newly recorded (n). PORTUGAL - Alentejo - SSMNP; 39¹8'05"N, 007²4'40"W; alt. 700 m; 19.XI.2018; Andrade J. leg.; S; Qp/Cs; M/A; UEVHFUNGI 2004329.

Identification. This species can be recognized by the blue-green, viscid, bell-shaped pileus, which at maturity becomes broadly convex to flat, darkening brown at the centre. Often fleeting remains of veil are present at the margins of the pileus. Lamellae adnate, crowded, pale grey at first, becoming purple-brown with age, with pale white margins. Stipe cylindrical, $2-6 \times 0.5-1.2 \mathrm{~cm}$, with a fleeting ring, whitish above the ring zone, darker bluegreen and covered with coarse white veil remains below the ring zone. Spores ellipsoidal, smooth, with a germ pore. It can be distinguished from Stropharia caerulea Kreisel, which is a much more common species having lamellae with brown margins.

Suillaceae

\section{Suillus bellinii (Inzenga) Kuntze}

Material examined. PORTUGAL - Alentejo - SSMNP; $39^{\circ} 17^{\prime} 10^{\prime \prime} \mathrm{N}, 007^{\circ} 20^{\prime} 57^{\prime \prime} \mathrm{W}$; alt. 770 m; 05.XI.2016; Andrade J. leg.; M; Ppi; M/A; UEVH-FUNGI 2003546.

\section{Suillus bovinus (L.) Roussel}

Material examined. PORTUGAL - Alentejo - SSMNP; $39^{\circ} 17^{\prime} 10^{\prime \prime} \mathrm{N}, 007^{\circ} 20^{\prime} 57^{\prime \prime} \mathrm{W}$; alt. $770 \mathrm{~m}$; 19.XI.2018; Andrade J. leg.; M; Ppi; M/A; UEVH-FUNGI 2004319.

Tremellaceae

\section{Tremella aurantia Schwein.}

Material examined. PORTUGAL - Alentejo - SSMNP; $39^{\circ} 23^{\prime} 44^{\prime \prime} \mathrm{N}, 007^{\circ} 25^{\prime} 09^{\prime \prime} \mathrm{W}$; alt. 580 m; 20.XI.2013; Andrade J. leg.; P; Qp/Qs; M/A; UEVH-FUNGI 2002342.

Tricholomataceae

\section{Arrhenia rickenii (Hora) Watling}

Material examined. Newly recorded (n). PORTUGAL - Alentejo • SSMNP; 39 23'44"N, 007²5'09"W; alt. 580 m; 12.XI.2018; Andrade J. leg.; S; Qp/Qs; M/A; UEVHFUNGI 2004410.

\section{Cantharellula umbonata (J. F. Gmel.) Singer \\ Figure $7 \mathrm{~F}$}

Material examined. Newly recorded (n). PORTUGAL - Alentejo - SSMNP; 39 $19^{\prime} 17^{\prime \prime} \mathrm{N}, 007^{\circ} 23^{\prime} 08^{\prime \prime} \mathrm{W}$; alt. 770 m; 27.XI.2016; Andrade J. leg.; S; Ppi; Atl.; UEVHFUNGI 2003678.

Identification. This species can be recognized by its convex to depressed pileus with a small, pointed umbo, incurved margin at first becoming upturned and wavy upon maturity, smooth to minutely hairy, grey to greyish-brown pileipellis. Crowded, decurrent, whitish lamellae, repeatedly and regularly forked, developing spot-like reddish or sometimes yellow stains when mature. Long, curved, and/or twisted stipe (always longer than the cap width), white to grey, silky above and usually with whitish mycelium near the base. Flesh becomes reddish when cut. This species can be distinguished from similar Cantharellus species, such as C. tubaeformis (Fr.) Quél., by having lamellae instead of forked veins. 


\section{Clitocybe bresadolana Singer}

Figure $7 \mathrm{G}$

Material examined. Newly recorded (n). PORTUGAL - Alentejo - SSMNP; 39¹8'05"N, 007²4'40"W; alt. 700 m; 08.XII.2017; Andrade J. leg.; S; Qp/Cs; Atl.; UEVHFUNGI 2003907.

Identification. This species can be recognized by its infundibuliform pileus with a rolled-up margin in young specimens, then recurved and sinuous, weakly punctuated, and striate in places. Pileipellis with appressed felt, whitish pink when wet, reddish brown when dry. White to cream lamellae often with reddish tinges, decurrent, wavy, and partially forked towards the base. Firm, fibrillose stipe, slightly thickened at base, brown-ochre, often covered with a white felt and adorned with fine rhizomorphs. Fruity or bitter almond-like smell. This species can be distinguished from C. glareosa Röllin \& Monthoux which is slenderer and is brighter reddish, and from Bonomyces sinopicus which has a characteristic mealy odour.

\section{Clitocybe costata Kühner \& Romagn.}

Material examined. PORTUGAL - Alentejo • SSMNP; 39²3'44"N, 007²5'09"W; alt. 580 m; 12.XI.2016; Andrade J. leg.; S; Qp/Qs; M/A; UEVH-FUNGI 2003572.

\section{Clitocybe fragrans (With.) P. Kumm.}

Material examined. PORTUGAL - Alentejo • SSMNP; $39^{\circ} 27^{\prime} 12^{\prime \prime} \mathrm{N}, 007^{\circ} 26^{\prime} 33^{\prime \prime} \mathrm{W}$; alt. 440 m; 05.XII.2016; Andrade J. leg.; S; Qp/Qs; Med.; UEVH-FUNGI 2003716.

\section{Clitocybe odora (Bull.) P. Kumm.}

Material examined. PORTUGAL - Alentejo • SSMNP; $39^{\circ} 23^{\prime} 44^{\prime \prime} \mathrm{N}, 007^{\circ} 25^{\prime} 09^{\prime \prime} \mathrm{W}$; alt. 580 m; 12.XI.2016; Andrade J. leg.; S; Qp/Qs; M/A; UEVH-FUNGI 2003594.

\section{Infundibulicybe geotropa (Bull.) Harmaja}

Material examined. PORTUGAL - Alentejo • SSMNP; $39^{\circ} 27^{\prime} 12^{\prime \prime} \mathrm{N}, 007^{\circ} 26^{\prime} 33^{\prime \prime} \mathrm{W}$; alt. $440 \mathrm{~m}$; 03.XI.2016; Andrade J. leg.; S; Qp/Qs; Med.; UEVH-FUNGI 2003725.

\section{Lepista nuda (Bull.) Cooke}

Material examined. PORTUGAL - Alentejo • SSMNP; $39^{\circ} 23^{\prime} 44^{\prime \prime} \mathrm{N}, 007^{\circ} 25^{\prime} 09^{\prime \prime} \mathrm{W}$; alt. 580 m; 03.XII.2016; Andrade J. leg.; S; Qp/Qs; M/A; UEVH-FUNGI 2003697.

\section{Lepista panaeolus (Fr.) P. Karst.}

Material examined. Newly recorded (n). PORTUGAL

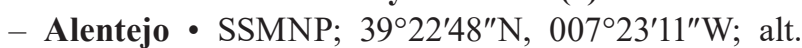
520 m; 30.X.2016; Andrade J. leg.; S; Ppn; Atl.; UEVHFUNGI 2003539.

\section{Melanoleuca polioleuca (Fr.) Kühner \& Maire Figure $7 \mathrm{H}$}

Material examined. Newly recorded (n). PORTUGAL - Alentejo - SSMNP; 39¹8'06" N, 007²4'10"W; alt. 700 m; 08.XII.2017; Andrade J. leg.; S; Ppi; M/A; UEVHFUNGI 2003897.
Identification. This species can be recognized by its greyish-brown, convex to depressed pileus with a broad umbo. Lamellae thin, crowded, whitish-cream. Cylindrical stipe with whitish fibrils on a dark background; reddish-brown flesh, darker towards the base of the stipe. Spindle-shaped cheilocystidia with crystals on the top. Melanoleuca melaleuca (Pers.) Murrill can be distinguished by its unchangeable flesh, and $M$. polioleuca (Fr.) Kühner \& Maire is darker coloured and with lageniform cystidia.

\section{Paralepista flacida (Sowerby) Vizzini}

Material examined. PORTUGAL - Alentejo • SSMNP; $39^{\circ} 19^{\prime} 15^{\prime \prime} \mathrm{N}, 007^{\circ} 24^{\prime} 47^{\prime \prime} \mathrm{W}$; alt. 600 m; 11.XI.2018; Andrade J. leg.; S; Qp/Qs; M/A; UEVH-FUNGI 2004498.

\section{Pseudoclitocybe cyathiformis (Bull.) Singer}

Material examined. PORTUGAL - Alentejo • SSMNP; $39^{\circ} 23^{\prime} 09^{\prime \prime} \mathrm{N}, 007^{\circ} 21^{\prime} 42^{\prime \prime} \mathrm{W}$; alt. 640 m; 06.I.2018; Andrade J. leg.; S; Qp/Qs; M/A; UEVH-FUNGI 2003931.

Tricholoma acerbum (Bull.) Quél.

Material examined. Newly recorded (n). PORTUGAL - Alentejo • SSMNP; $39^{\circ} 18^{\prime} 05^{\prime \prime} \mathrm{N}, 007^{\circ} 24^{\prime} 40^{\prime \prime} \mathrm{W}$; alt. 700 m; 14.XI.2016; Andrade J. leg.; M; Qp/Cs; M/A; UEVHFUNGI 2003615.

\section{Tricholoma columbetta (Fr.) P. Kumm.}

Figure 7I

Material examined. Newly recorded (n). PORTUGAL - Alentejo • SSMNP; $39^{\circ} 18^{\prime} 15^{\prime \prime} \mathrm{N}, 007^{\circ} 24^{\prime} 35^{\prime \prime} \mathrm{W}$; alt. 720 m; 05.XI.2016; Andrade J. leg.; M; Qp/Cs; M/A; UEVHFUNGI 2003567.

Identification. This species can be recognized by the pure white colour of its basidiocarps. Pileus is convex to applanate at maturity, up to $10 \mathrm{~cm}$ in diameter, with a slightly curved, wavy margin, smooth and pure white. Lamellae are white, crowded, and sinuate. Stipe fibrillose, compact, sometimes curved, smooth, white with blue hues near the base. Spores are ellipsoidal and smooth. Can be easily discerned from Calocybe gambosa (Fr.) Donk by the absence of mealy odour.

\section{Tricholoma equestre (L.) P. Kumm.}

Material examined. PORTUGAL - Alentejo - SSMNP; $39^{\circ} 19^{\prime} 56^{\prime \prime} \mathrm{N}, 007^{\circ} 22^{\prime} 52^{\prime \prime} \mathrm{W}$; alt. 710 m; 03.XII.2016; Andrade J. leg.; M; Ppi; M/A; UEVH-FUNGI 2003708.

\section{Tricholoma imbricatum (Fr.) P. Kumm.}

Figure 7J

Material examined. Newly recorded (n). PORTUGAL - Alentejo • SSMNP; 39 $19^{\prime} 56^{\prime \prime} \mathrm{N}, 007^{\circ} 22^{\prime} 52^{\prime \prime} \mathrm{W}$; alt. 710 m; 03.XII.2016; Andrade J. leg.; M; Ppi; M/A; UEVHFUNGI 2003701.

Identification. This species can be recognized by the convex to applanate pileus, $6-15 \mathrm{~cm}$ in diameter, margin at first inrolled then decurved, often wavy at maturity; surface dry, brown, fading near the margin, fibrillose to 
finely scaled or cracked near the margin. Lamellae close, adnexed to notched, cream colour with darker brown spots at maturity. Stipe solid, sometimes club-shaped or tapering; surface dry, dull, cream-buff, developing brown stains below. Spores broadly ellipsoid and smooth. Tricholoma vaccinum (Schaeff.) P. Kumm. is morphologically similar, but does not occur in conifer stands, the pileus is reddish, and the stipe is hollow.

\section{Tricholoma populinum J. E. Lange}

Material examined. Newly recorded (n). PORTUGAL - Alentejo • SSMNP; 39²2'38"N, 007 23'13"W; alt. 520 m; 11.XII.2016; Andrade J. leg.; M; Ppn; Atl.; UEVHFUNGI 2003726.

\section{Tricholoma portentosum (Fr.) Quél.}

Material examined. PORTUGAL - Alentejo • SSMNP; $39^{\circ} 19^{\prime} 56^{\prime \prime} \mathrm{N}, 007^{\circ} 22^{\prime} 52^{\prime \prime} \mathrm{W}$; alt. 710 m; 03.XII.2016; Andrade J. leg.; M; Ppi; M/A; UEVH-FUNGI 2003695.

\section{Tricholoma scalpturatum (Fr.) Quél.}

Figure $7 \mathrm{~K}$

Material examined. Newly recorded (n). PORTUGAL - Alentejo - SSMNP; $39^{\circ} 27^{\prime} 12^{\prime \prime} \mathrm{N}, 007^{\circ} 26^{\prime} 33^{\prime \prime} \mathrm{W}$; alt. 440 m; 05.XI.2016; Andrade J. leg.; M; Qp/Qs; Med; UEVHFUNGI 2003583.

Identification. This species can be identified by the convex to applanate pileus, up to $7-8 \mathrm{~cm}$ in diameter, with dense overlapping scales, which are dark brown in the centre fading to greyish colour towards the margin. Margin curved, then wavy with prominent greyish scales. Lamellae crowded, sinuate, even edges and ash-grey to nearly white in age. Stem cylindrical, straight, solid to hollow in age, finely fibrous, often with a faint stem ring zone created by a fleeting veil, white or pale grey. Spores ellipsoidal and smooth. Strong mealy odour; this differs from T. terreum (Schaeff.) P. Kumm., which lacks the strong mealy odour and exhibits a uniformly greyish-black pileus. Tricholoma cingulatum (Almfelt ex Fr.) Jacobashch has a well-defined and permanent ring zone, and T. sciodes (Pers.) C. Martín has violet hues in the pileus and black speckles in the margin of the lamellae.

\section{Tricholoma stans (Fr.) Sacc.}

Figure 7L

Material examined. Newly recorded (n). PORTUGAL - Alentejo - SSMNP; 39 $19^{\prime} 56^{\prime \prime} \mathrm{N}, 007^{\circ} 22^{\prime} 52^{\prime \prime} \mathrm{W}$; alt. 710 m; 03.XII.2016; Andrade J. leg.; M; Ppi; M/A; UEVHFUNGI 2003689.

Identification. This species can be recognized by the convex to almost applanate pileus, $5-10 \mathrm{~cm}$ in diameter, smooth, reddish-brown to grey-brown with age. Margin paler than the centre of the pileus, sometimes striate. Lamellae cream colour, smooth, crowded, notched and with dark speckles throughout. Stipe cylindrical, smooth, or finely fibrillose longitudinally, initially white then pale brown. Flesh white, darkening brown when bruised. Spores ellipsoidal and smooth. It can be distinguished from T. pessundatum (Fr.) Quél. by the lack of a strong mealy odour and taste.

\section{Tricholoma sulphureum (Bull.) P. Kumm.}

Material examined. PORTUGAL - Alentejo - SSMNP; $39^{\circ} 08^{\prime} 04^{\prime \prime} \mathrm{N}, 007^{\circ} 12^{\prime} 37^{\prime \prime} \mathrm{W}$; alt. 370 m; 09.XI.2015; Andrade J. leg.; M; Qs/Qr; M/A; UEVH-FUNGI 2003066.

\section{Tricholomopsis rutilans (Schaeff.) Singer}

Material examined. PORTUGAL - Alentejo - SSMNP; $39^{\circ} 18^{\prime} 06^{\prime \prime} \mathrm{N}, 007^{\circ} 24^{\prime} 10^{\prime \prime} \mathrm{W}$; alt. 700 m; 01.XI.2014; Andrade J. leg.; S; Ppi; M/A; UEVH-FUNGI 2002784.

\section{Discussion}

Despite the national recognition of the biological importance of SSMNP as a Site of Community Importance under Nature 2000 network, this study is the first extended macrofungal survey ever performed in the park. We show that the SSMNP hosts an outstandingly species rich and diverse macrofungal assemblage, similar to other protected areas in Portugal (Natário et al. 2019). However, when compared with other Quercus spp. stands (holm oak and cork oak) within or near protected areas (Santos-Silva et al. 2011; Santos-Silva and Louro 2016; Pinto-Correia et al. 2018), the SSMNP macrofungal communities are richer and more diverse.

Regarding the representativeness of trophic guilds in the park, the high relative percentage $(60 \%)$ of mycorrhizal species can be attributed to the multitude of plant hosts available in mixed stands. Plant diversity and fungal host specificity are known to have a positive impact on ectomycorrhizal fungi diversity, (Kernaghan et al. 2003; Jumpponen et al. 2012; Dickie et al. 2013).

Most notably, the majority of the macrofungal taxa occurred more frequently in Q. pyrenaica stands. In fact, $Q$. pyrenaica have a great importance to the landscapes of the Iberian Peninsula, forming highly biodiverse forest ecosystems which are protected by European legislation. These forests have been seriously deteriorated as a result of historical human actions and frequent replacement with more profitable tree species, such as pine or eucalyptus (Calvo et al. 1999; Tárrega et al. 2006). Our work clearly indicates that $Q$. pyrenaica stands are formidably diverse in terms of macrofungi, hosting extremely rare taxa, such as, Cortinarius xanthophyllus, Cantharellus melanoxeros, Rheubarbariboletus persicolor, and Russula sericatula. Therefore, the loss of these stands poses a great threat to the maintenance and conservation of these rich and diverse macrofungal communities.

It is no secret that the majority of protected areas (natural parks and reserves) under the Nature 2000 network were created based on animal or plant data, or due to the presence of particular habitats. Fungi are often poorly understood and have been universally overlooked in planning and preparation of conservation plans (SennIrlet et al. 2007). It is vital to reverse this tendency, otherwise millions of species could be endangered. Recently, 
the IUCN developed a huge effort to launch the first list of fungi conservation status, but Mediterranean taxa are still poorly represented. In that context, we report in the SSMNP the presence of one threatened species included in the Global Fungal Red List, Tricholoma acerbum (Bull.) Quél., which is classified as Vulnerable. Poronia punctata (L.) Fr., Agaricus arvensis Schaeff., Agaricus campestris L., Coprinus comatus (O. F. Müll.) Pers., Lycoperdon perlatum Pers., Amanita caesarea (Scop.) Pers., Cantharellula umbonata (J. F. Gmel.) Singer, Boletus reticulatus Schaeff., and Hydnum repandum L. are also included in the Global Fungal Red List, but all of which are classified as Least Concern.

Our work represents an important contribution to the knowledge of the macrofungal community of the SSMNP. It also reinforces the importance of mixed forest stands on macrofungal conservation, particularly of mycorrhizal taxa. Having brought to light the high diversity and richness of this group, we hope that the Portuguese national conservation organizations and park authorities begin to pay due attention to macrofungal communities and, in time, will consider them in conservation management plans.

\section{Acknowledgements}

We thank Dr. Tânia Nobre for editing and revising the English language. We also wish to thank the journal's editorial team for their assistance throughout the manuscript submission process. We are also grateful to the anonymous reviewers for their valuable suggestions and thoughtful reviews of the manuscript.

Funding. We acknowledge National Funds through FCT - Foundation for Science and Technology under the Project UIDB/05183/2020.

\section{Authors' Contributions}

Conceptualization: JA, CSS. Data curation: RL, CSS, BAFN. Formal analysis: CSS. Funding acquisition: CSS, RL. Investigation: CSS, JA, RL, BAFN. Methodology: RL, CSS, JA. Project administration: CSS. Resources: CSS. Supervision: RL. Writing - original draft: JA, BAFN, CSS, RL. Writing - review and editing: RL, CSS.

\section{References}

Bon M (2004) Champignons de France et d'Europe occidentale. Flammarion, Paris, France, 368 pp.

Breitenbach J, Kränzlin F (1981) Champignons de Suisse, Tome 1: les Ascomycètes. Edition Mykologia, Lucerne, Switzerland, 310 pp.

Breitenbach J, Kränzlin F (1986) Champignons de Suisse, tome 2: champignons sans lames. Edition Mykologia, Lucerne, Switzerland, $412 \mathrm{pp}$.

Breitenbach J, Kränzlin F (1991) Champignons de Suisse, tome 3: bolets et champignons à lames, 1ère partie. Edition Mykologia, Lucerne, Switzerland, 364 pp.

Breitenbach J, Kränzlin F (1995) Champignons de Suisse, tome 4: champignons à lames, 2ème partie. Edition Mykologia, Lucerne,
Switzerland, $371 \mathrm{pp}$.

Breitenbach J, Kränzlin F (2000) Champignons de Suisse, tome 5: champignons à lames, 3ème. Edition Mykologia, Lucerne, Switzerland, $340 \mathrm{pp}$.

Cabral MJ, Almeida J, Almeida PR, Delliger T, Almeida NF, Oliveira ME, Palmeirim JM, Queirós AI, Rogado L, Santos-Reis M (2005). Livro Vermelho dos vertebrados de Portugal. Instituto da Conservação da Natureza, Lisboa, Portugal, 659 pp.

Calonge FD (1998) Flora mycologica iberica, vol. 3: Gasteromycetes, I. Lycoperdales, Nidulariales, Phallales, Sclerodermatales, Tulostomatales. J. Cramer, Suttgart, Germany, 272 pp.

Calvo L, Tárrega R, de Luis E (1999) Post-fire succession in two Quercus pyrenaica communities with different disturbance histories. Annals of Forest Science 56: 441-447. https://doi.org/10.1051/ forest: 19990508

Castro-Antunes JS (1996) Aspectos sinfitossociológicos da Serra de S. Mamede. Silva Lusitana 4: 97-107.

Costa JC, Aguiar C, Capelo JH, Lousã M, Neto C (1998) Biogeografia de Portugal continental. Quercetea 0: 5-56.

Dickie IA, Martínez-García LB, Koele N, Grelet GA, Tylianakis JM, Peltzer DA, Richardson SJ (2013) Mycorrhizas and mycorrhizal fungal communities throughout ecosystem development. Plant and Soil 367: 11-39. https://doi.org/10.1007/s11104-013-1609-0

Ferreira AM (2000) Caracterização de Portugal continental. Dados geoquímicos de base de sedimentos fluviais de amostragem de baixa densidade de Portugal Continental: estudo de factores de variação regional. $\mathrm{PhD}$ thesis, Aveiro University, Aveiro, Portugal, 234 pp.

Flora-On (Flora de Portugal Interactiva) (2022) Sociedade Portuguesa de Botânica. Portugal. https://flora-on.pt/index.php\#1serra+de+ s\%C3\%A3o+mamede. Accessed on: 2022-1-21.

Frade B, Alfonso A (2003) Atlas fotográfico de los hongos de la Península Ibérica. Celarayn, León, Spain, 547 pp.

GBIF (Global Biodiversity Information Facility) (2021) Copenhagen, Denmark. https://www.gbif.org. Accessed on: 2021-4-1.

Gerhardt E, Vila J, Llimona X (2000) Hongos de España y de Europa. Omega, Barcelona, Spain, 957 pp.

ICNF (2003) Plano de Ordenamento do Parque Natural da Serra de S. Mamede. Instituto da Conservação da Natureza e das Florestas, Lisboa, Portugal. http://www2.icnf.pt/portal/pn/biodiversidade/ ordgest/poap/popnssm/plano-de-ordenamento-do-parquenatural-da-serra-de-s-mamede-documentos. Accessed on: 2022$1-21$.

IUCN (International Union for Conservation of Nature) (2022) The IUCN Red List of threatened species. Version 2021-3. https:// www.iucnredlist.org. Accessed on: 2022-1-21.

Jumpponen A, Brown SP, Trappe JM, Cázares E, Strömmer R (2012) Twenty years of research on fungal-plant interactions on Lyman Glacier forefront-lessons learned and questions yet unanswered. Fungal Ecology 5: 430-442. https://doi.org/10.1016/j. funeco.2012.01.002

Kernaghan G, Widden P, Bergeron Y, Légaré S, Paré D (2003) Biotic and abiotic factors affecting ectomycorrhizal diversity in boreal mixed-woods. Oikos 102: 497-504.

Kränzlin F (2005) Champignons de Suisse, ome 6: Russulaceae. Edition Mykologia, Lucerne, Switzerland, 319 pp.

Malkmus R (1997) Die Verbreitung der Amphibien und Reptilien in der Serra de São Mamede, Portugal. Zeitschrift für Feldherpetologie 4: 63-92.

Marta-Pedroso C, Laporta L, Gama I, Domingos T (2018) Economic valuation and mapping of Ecosystem Services in the context of protected area management (Natural Park of Serra de São Mamede, Portugal). One Ecosystem 3: e26722. https://doi.org/10.3897/ oneeco.3.e26722

Moreno G, Manjón JL (2010) Guía de hongos de la Península Ibérica. Omega, Barcelona, Spain, 1417 pp.

Natário B, Louro R, Santos-Silva C (2019) Macrofungi of Mata da Margaraça (Portugal), a relic from the Tertiary Age. Biodiversity 
Data Journal 7: e38177. https://doi.org/10.3897/bdj.7.e38177

Oliveira J (2014) Marvão-estudos e documentos de apoio à candidatura a Património Mundial. Edições Colibri, Marvão, Portugal, 272 pp.

Pegler DN, Spooner B, Læssøe T (1995) British puffballs, earthstars and stinkhorns: an account of the British gasteroid fungi. Royal Botanic Gardens, Kew, UK, 255 pp.

Pinto-Correia T, Guiomar N, Ferraz-de-Oliveira MI, Sales-Baptista E, Rabaça J, Ribeiro N, Sá-Sousa P, Santos P, Santos-Silva C, Simões MP, Belo A, Godinho S, Azeda C, Almeida M, Louro R, Vaz M, Godinho C, Lopes-de-Castro J, Silvestre M, Gomes L, Costa P, Fonseca E, Catarino L, Costa D (2018) Progress in identifying high nature value montados: impacts of grazing on hardwood rangeland biodiversity. Rangeland Ecology and Management 71 (5): 612-625. https://doi.org/10.1016/j.rama.2018.01.004

Quintano P, Caudullo G, Rigo D (2016) Quercus pyrenaica in Europe: distribution, habitat, usage and threats. In: Ayanz SM, Rigo D, Caudullo G, Durrant T, Mauri A (Eds.) European atlas of forest tree species, Publication Office of the European Union, Luxembourg, e01f807. https://ies-ows.jrc.ec.europa.eu/efdac/download/ Atlas/pdf/Quercus_pyrenaica.pdf. Accessed on: 2022-1-21.

Roux P (2006) Mille et un champignons. Roux, Saint-Sigolène, France, 1224 pp.

Santos-Silva C, Louro R (2016) Assessment of the diversity of epigeous Basidiomycota under different soil-management systems in a Montado ecosystem: a case study conducted in Alentejo.
Agroforest. Syst. 90: 117-126. https://doi.org/10.1007/s10457-0159800-3

Santos-Silva C, Gonçalves A, Louro R (2011) Canopy cover influence on macrofungal richness and sporocarp production in montado ecosystems. Agroforestry Systems 82: 149-159. https://doi. org/10.1007/s10457-011-9374-7

Sarnari M (1998) Monografia illustrata del genere Russula in Europa, Tomo Primo. Associazione Micologica Bresadola, Trento, Italy, 799 pp.

Sarnari M (2005) Monografia illustrata del genere Russula in Europa, tomo secondo. Associazione Micologica Bresadola, Trento, Italy, 769 pp.

Senn-Irlet B, Heilmann-Clausen J, Genney D, Dahlberg A (2007). Guidance for conservation of macrofungi in Europe. Document prepared for the European Council for Conservation of Fungi (ECCF) within the European Mycological Association (EMA) and the Directorate of Culture and Cultural and Natural Heritage, Council of Europe, Strasbourg, France, 39 pp.

Sérgio C, Cros R, Brugués M, Casas C (1997) Flora e Vegetação briológica do Parque Natural da Serra de São Mamede. Portugaliae Acta Biológica, Sér. B, Sistemática 17: 5-46.

Tárrega R, Calvo L, Marcos E, Taboada A (2006) Forest structure and understory diversity in Quercus pyrenaica communities with different human uses and disturbances. Forest Ecology and Management 227: 50-58. https://doi.org/10.1016/j.foreco.2006.02.008 